\title{
PRIMEIRA VARIAÇÃO DA \\ ENERGIA E GEODÉSICAS NA \\ GEOMETRIA SUB-RIEMANNIANA
}

José Carlos Simon de Miranda

DISSERTAÇÃO APRESENTADA

$\mathrm{AO}$

INSTITUTO DE MATEMÁTICA E ESTATÍSTICA

DA

UNIVERSIDADE DE SÃO PAULO

PARA

OBTENÇÃO DO GRAU

$\mathrm{DE}$

MESTRE EM MATEMÁTICA

Área de Concentração: Geometria

Orientador: Prof. Dr. José Antonio Verderesi

- São Paulo, maio de 1998 - 


\section{PRIMEIRA VARIAÇÃO DA ENERGIA E GEODÉSICAS NA GEOMETRIA SUB-RIEMANNIANA}

Este exemplar corresponde à redação final da dissertação apresentada por José Carlos Simon de Miranda, devidamente corrigida e aprovada pela Comissão Julgadora

São Paulo, 15 de maio de 1998.

\section{Comissão Julgadora}

- Prof. Dr. José Antonio Verderesi - IME-USP

- Prof. Dr. Francisco Mercuri - IMECC-UNICAMP

- Prof. Dr. Nicolai Gusevskii - UFMG 


\section{Resumo}

Neste trabalho demonstramos a fórmula da primeira variação da energia para variedades sub-riemannianas de contato e deduzimos desta a equação das geodésicas para tais variedades.

No primeiro capítulo definimos variedade sub-riemanniana de contato e apresentamos um teorema sobre a existência e unicidade de uma derivada covariante associada à estrutura sub-riemanniana.

No segundo capítulo estudamos os pontos críticos do funcional energia definido no espaço das curvas de contato e demonstramos um teorema que caracteriza as geodésicas sub-riemannianas.

Finalmente, no terceiro capítulo, apresentamos o cálculo das geodésicas no espaço de Heisemberg, na esfera $S^{3}$ e na quádrica $\mathbb{Q}^{3}$. 


\section{Abstract}

In this work we derive the formula of the first variation of energy for contact subriemannian manifolds and from it, we deduce the equation of geodesics for such manifolds.

First chapter is devoted to define contact sub-riemannian manifold and to present a theorem about the existence and uniqueness of a covariant derivative associated to the sub-riemannian structure.

In chapter two we study the critical points of the energy functional defined on the space of contact curves. A characterization theorem for sub-riemannian geodesics is also shown.

Finally, in chapter three, we derive the equations of geodesics in $H^{3}, S^{3}$ and $\mathbb{Q}^{3}$. 
"São lindos

Seus olhos verdes são lindos

Muito, muito mesmo!

É impressionante!"

Lindos quando iluminados pelos teus.

E era verdade. A única no momento.

O céu brilhante da montanha

Imita seus olhos.

A mais branca e jovem rosa

Inveja sua pele.

Os campos de trigo seu cabelo.

A lua crescente seu sorriso.

Moça dos olhos brilhantes de luz do fogo

Quando encontrar sorrindo seus pingos de céu Vou transformar o momento em vida

De fogo da terra e azul do mar. 


\section{Agradecimentos}

Agradeço ao Prof. Dr. José Antônio Verderesi pela ótima orientação.

Ao amigo Verderesi. Este trabalho não existiria sem sua ajuda.

Aos meus pais pelo amor, carinho e dedicação.

Aos amigos Marcelo e Yamin pelo companheirismo.

À Zara e ao Daciberg pela ajuda e incentivo. 
Para meu pai.

De quem recebi a honestidade a coragem, o amor e a bondade. 


\section{Conteúdo}

Introdução 1

1 Variedades sub-riemannianas de contato 3

$\begin{array}{lll}2 & \text { Variação da energia } & 17\end{array}$

3 Exemplos 44

Exemplo 1: Espaço de Heisenberg . . . . . . . . . . . . . . . . . . . . . . . . 44

Exemplo 2: a esfera $S^{3} \ldots \ldots \ldots \ldots \ldots \ldots$. . . . . . . . . . . . . . 51

Exemplo 3: a quádrica $\mathrm{Q}^{3} \ldots \ldots \ldots \ldots \ldots$

$\begin{array}{ll}\text { Referências } & 65\end{array}$ 


\section{Introdução}

Uma variedade sub-riemanniana de contato é uma variedade na qual temos uma distribuição de contato e uma métrica definida na distribuição.

Utilizamos o Capítulo 1 para apresentar as definições de distribuições de contato, variedade sub-riemanniana de contato e forma de contato; e enunciar proposições relacionadas a estes conceitos. Ainda no Capítulo 1, demonstramos um teorema de existência e unicidade de uma derivada covariante associada à estrutura sub-riemanniana e apresentamos também a primeira equação de estrutura.

No segundo capítulo apresentamos o funcional energia e deduzimos a fórmula da primeira variação da energia. Com base na primeira variação da energia, deduzimos um teorema que caracteriza as geodésicas em variedades sub-riemannianas de contato.

Em [11] Strichartz deduz as equações das geodésicas para variedades sub-riemannianas não necessariamente de contato e enuncia um teorema de caracterização de geodésicas através destas equações. Entretanto, Montgomery [9] mostrou que tal resultado era falso, apresentando contra exemplos de curvas extremais do funcional energia que não satisfazem às equações das geodésicas propostas por Strichartz. Posteriormente, Rumin [10] caracterizou, através de equações, as geodésicas em variedades pseudo hermitianas de contato. Entretanto, o resultado por ele apresentado está incompleto pois depende da existência de variações de contato com extremos fixos cujas variações infinitesimais são por ele utilizadas. Demonstramos a existência de tais variações, na Proposição 2.7, e deduzimos o teorema de caracterização de geodésicas para variedades sub-riemannianas de 
contato. Terminamos o segundo capítulo demonstrando que as geodésicas em variedades sub-riemannianas de contato são suaves.

O último capítulo é dedicado à aplicação destes teoremas a três exemplos de variedades sub-riemannianas de contato de dimensão três, o espaço de Heisenberg, a esfera $S^{3}$ e a quádrica $\mathbb{Q}^{3}$ que, na geometria riemanniana, correspondem aos espaços de curvatura constante nula, positiva e negativa. Nestes três exemplos também determinamos a aplicação exponencial em pontos particulares. Nestes exemplos fazemos uso dos conceitos do primeiro capítulo, sendo que nos segundo e terceiro exemplos utilizamos variáveis complexas para obtermos os resultados de uma maneira mais direta.

Boa leitura! 


\section{Capítulo 1}

\section{Variedades sub-riemannianas de contato}

Neste capítulo definiremos variedades sub-riemannianas de contato e estudaremos uma derivada covariante a elas associdada.

Os objetos estudados, quando aplicável, serão todos de classe $C^{\infty}$ a menos de menção contrária.

Seja $M$ uma variedade e TM o fibrado tangente.

Uma distribuição $D$ é um subfibrado de TM e a ela associaremos uma métrica que chamaremos de $g$.

Serão consideradas distribuições de codimensão 1 transversalmente orientáveis.

Proposição 1.1 Se M é uma variedade e D uma distribuição tranversalmente orientável de codimensão 1, então existe um campo transverso $U$ definido em $M$ que não se anula.

Tomemos em $M$ uma métrica $g_{1}$.

Seja $\left\{V_{i}\right\}, i \in I$, uma cobertura por abertos de $M$. Como $D$ é transversalmente orientável, existe um campo de retas orientável sobre $M$. Assim, em cada aberto $V_{i}$ temos um campo de vetores $U_{i}$ que não se anula de tal forma que na intersecção de dois abertos $V_{i} \cap V_{j}$ os campos $U_{i}$ e $U_{j}$ são da forma $U_{i}=\alpha U_{j}$, onde $\alpha: U_{i} \cap U_{j} \rightarrow \mathbb{R}_{+}^{*}$.

Seja $N_{i}$ o campo definido em $V_{i}$ por $N_{i}=\frac{U_{i}}{\left\|U_{i}\right\|}$. 
Se $p \in V_{i} \cap V_{j}$, então

$$
N_{i}(p)=\frac{U_{i}(p)}{\left\|U_{i}(p)\right\|}=\frac{\alpha U_{j}(p)}{\left\|\alpha U_{j}(p)\right\|}=\frac{U_{j}(p)}{\left\|U_{j}(p)\right\|}=N_{j}(p) .
$$

Definimos $U$ sobre $M$ por $U(p)=N_{k}(p)$, onde $k \in\left\{i \in I \mid p \in V_{i}\right\}$.

Proposição 1.2 Se $M$ é uma variedade e D uma distribuição transversalmente orientável de codimensão 1, então existe uma 1-forma $\eta$ definida em $M$ tal que $\operatorname{ker} \eta=D$.

Seja $U$ o campo transverso da Proposição 1.1.

$$
T_{p} M=[U(p)] \oplus D_{p}
$$

Sendo $V$ um vetor de $T_{p} M$ ele é escrito de maneira única como

$$
V=v_{t} U_{t}(p)+v_{d}, \quad v_{t} \in \mathbb{R} \quad \text { e } \quad v_{d} \in D_{p} .
$$

Definimos $\eta$ por $\eta_{p}(V)=v_{t}$.

Utilizando as notações das proposições anteriores, podemos montar o diagrama comutativo

$$
\begin{aligned}
& T M \quad \stackrel{\eta}{\longrightarrow} \quad \mathbb{R} \\
& \downarrow \pi_{1} \quad \uparrow \pi_{2} \\
& T M / D \stackrel{\phi}{\longrightarrow} \quad M \times \mathbb{R} \\
& (p, V) \in T M \\
& \pi_{1}(p, V)=\left(p, v_{t} U(p) \bmod D_{p}\right) \\
& \phi\left(p, v_{t} U(p) \bmod D_{p}\right)=\left(p, v_{t}\right) \\
& \pi_{2}\left(p, v_{t}\right)=v_{t} \\
& \eta(p, V)=v_{t}
\end{aligned}
$$

Assim temos as identificações $T M / D \cong M \times \mathbb{R}$ e $(T M / D)_{p} \cong \mathbb{R}$. 
Definição 1.1 Uma variedade sub-riemanniana de codimensão 1 é uma variedade $M$ munida de uma distribuição $D$, de codimensão 1 e de uma métrica g em $D$.

Indicaremos esta variedade pela terna $(M, D, g)$ ou mais diretamente por $M$.

Vamos denotar campos tangentes à distribuição $D$ pelas letras $X, Y$ e $Z$ e campos tangentes a $M$ por $U, V$ e $W$. Utilizamos a notação $X \in D$ e $U \in T M$.

Consideremos agora a aplicação bilinear anti-simétrica definida por

$$
\begin{aligned}
L: D \times D & \rightarrow T M / D \\
(X, Y) & \rightarrow L(X, Y)=[X, Y] \bmod D
\end{aligned}
$$

Sejam $p \in M, U$ um aberto de $M$ com $p \in U$ e $f, g: U \rightarrow \mathbb{R}$

$$
\begin{aligned}
{[f X, Y] g } & =f X(Y g)-Y(f X) g=f X Y g-f Y X g-(Y f) X g \\
& =f[X, Y] g-(Y f) X g=(f[X, Y]-(Y f) X) g
\end{aligned}
$$

$(Y f) X \in D$. Assim,

$$
\begin{aligned}
& L(f X, Y)=f L(X, Y) \\
& X=\sum_{i} X^{i} \frac{\partial}{\partial X_{i}}, \quad Y=\sum_{j} Y^{j} \frac{\partial}{\partial X_{j}} \\
& L(X, Y)=\sum_{i, j} X^{i} Y^{j} L\left(\frac{\partial}{\partial X_{i}}, \frac{\partial}{\partial X_{j}}\right) .
\end{aligned}
$$

Sendo $X_{1}, X_{2}, Y_{1}, Y_{2} \in D, \operatorname{com} X_{1}(p)=X_{2}(p)$ e $Y_{1}(p)=Y_{2}(p)$, temos

$$
\begin{aligned}
L_{p}\left(X_{1}, Y_{1}\right) & =\sum_{i, j} X_{1}^{i}(p) Y_{1}^{j}(p) L_{p}\left(\frac{\partial}{\partial X_{i}}, \frac{\partial}{\partial X_{j}}\right) \\
& =\sum_{i, j} X_{2}^{i}(p) Y_{2}^{j}(p) L_{p}\left(\frac{\partial}{\partial X_{i}}, \frac{\partial}{\partial X_{j}}\right)=L_{p}\left(X_{2}, Y_{2}\right) .
\end{aligned}
$$

Portanto, o valor de $L$ só depende do valor dos campos no ponto $p$ e assim para cada $p \in M, L_{p}: D_{p} \times D_{p} \rightarrow(T M / D)_{p}$ está bem definida. 
Definição 1.2 Uma distribuição de contato sobre $M$ é uma distribuição $D$ de codimensão 1 tal que L é não degenerada.

Definição 1.3 Uma variedade sub-riemanniana $M$ se diz de contato se $D$ é de contato.

Proposição 1.3 A dimensão de uma variedade sub-riemanniana de contato é ímpar.

Seja $A$ a representação matricial da aplicação $L$ e $k$ a dimensão de $D$.

Como $L$ é não degenerada, $\operatorname{det} A \neq 0$.

Como $L$ é anti-simétrica, temos

$$
\begin{aligned}
& A+A^{t}=0 \\
& A=-A^{t} \rightarrow \operatorname{det} A=(-1)^{k} \operatorname{det} A^{t}=(-1)^{k} \operatorname{det} A \rightarrow k=2 n .
\end{aligned}
$$

Portanto, $\operatorname{dim} D=2 n$ e $\operatorname{dim} M=2 n+1$.

Seja $\left\{e_{i}\right\}, 1 \leq i \leq 2 n$, uma base local ortonormal de $D,\left\{e_{i}, f\right\}$ uma base local de $T M$ e $\left\{\theta^{i}, \varphi\right\}$ sua base dual.

Definição 1.4 A $2 n$-forma sobre $M$ que assume valor 1 quando calculada em qualquer base ortonormal positiva de $D$ é chamada de elemento de volume em D.

Utilizamos a notação $\mathrm{d} V$ para a forma elemento de volume em $D$. Localmente podemos escrever $\mathrm{d} V=\theta^{1} \wedge \cdots \wedge \theta^{2 n}=\bigwedge_{i=1}^{2 n} \theta^{i}$.

Proposição 1.4 Existe uma única 1-forma $\omega$ sobre $M$, a menos de sinal, tal que ker $\omega=$ $D$ e d $\left.\omega\right|_{D} ^{n}=2^{n} n ! \mathrm{d} V$.

Seja $\eta$ uma 1-forma sobre $M \operatorname{com} \operatorname{ker} \eta=D$ (Proposição 1.2).

$$
\begin{aligned}
\mathrm{d} \eta(X, Y) & =X \eta(Y)-Y \eta(X)-\eta([X, Y]) \\
X, Y \in D & \rightarrow \eta(X)=\eta(Y)=0 \\
\mathrm{~d} \eta(X, Y) & =-\eta([X, Y]) .
\end{aligned}
$$


Como $L(X, Y)=[X, Y] \bmod D$ é não degenerada, $\left.\mathrm{d} \eta\right|_{D}$ é não degenerada.

Sendo $\left.\mathrm{d} \eta\right|_{D}$ não degenerada, existe uma base $\left\{b_{i}\right\}, 1 \leq i \leq 2 n$, local de $D$ com base dual $\left\{\eta_{i}\right\}, 1 \leq i \leq 2 n$, onde escrevemos

$$
\begin{aligned}
\left.\mathrm{d} \eta\right|_{D} & =\sum_{i=1}^{n} \eta_{2 i-1} \wedge \eta_{2 i} \\
\left.\mathrm{~d} \eta\right|_{D} ^{n} & =\left(\sum_{i=1}^{n} \eta_{2 i-1} \wedge \eta_{2 i}\right)^{n}=n ! \bigwedge_{i=1}^{2 n} \eta_{i}
\end{aligned}
$$

Logo, $\left.\mathrm{d} \eta\right|_{D} ^{n}$ é não degenerada.

Portanto existe $f: M \rightarrow \mathbb{R} \bigwedge_{i=1}^{2 n} \eta_{i}=f . \mathrm{d} V$, onde $f: M \rightarrow \mathbb{R}$ pode ser escolhida positiva.

Consideremos a forma $g \eta$, onde $g: M \rightarrow \mathbb{R}$,

$$
\begin{aligned}
\mathrm{d}(g \eta) & =\mathrm{d} g \wedge \eta+g \mathrm{~d} \eta \\
\left.\mathrm{d}(g \eta)\right|_{D} & =\left.(\mathrm{d} g \wedge \eta+g \mathrm{~d} \eta)\right|_{D}=\left.g \mathrm{~d} \eta\right|_{D}, \text { pois ker } \eta=D \\
\left.\mathrm{~d}(g \eta)\right|_{D} ^{n} & =g^{n}\left(\left.\mathrm{~d} \eta\right|_{D} ^{n}\right)=g^{n} n ! f \mathrm{~d} V \\
g^{n} f n ! \mathrm{d} V & =2^{n} n ! \mathrm{d} V \leftrightarrow g^{n} f=2^{n} \leftrightarrow g=2 f^{(-1 / n)} .
\end{aligned}
$$

Portanto, a forma procurada é $\omega=2 f^{(-1 / n)} \eta$.

Definição 1.5 A forma $\omega$ da proposição anterior é chamada de forma de contato da variedade sub-riemanniana.

Proposição 1.5 Existe um único campo $\xi$ sobre $M$ com as propriedades $\omega(\xi)=1 e$ $i_{\xi} \mathrm{d} \omega=0$.

Seja $U$ tal que $\omega(U)=1$.

$i_{U} \mathrm{~d} \omega(W)=\mathrm{d} \omega(U, W)$ é uma 1 -forma em $M$. Tomemos sua restrição a $D,\left.i_{U} \mathrm{~d} \omega\right|_{D}$, que é uma forma linear sobre $D$

$$
i_{U} \mathrm{~d} \omega_{D}(Y)=\mathrm{d} \omega(U, Y) .
$$


Como d $\omega$ é não degenerada sobre $D$, existe um único $Z$ campo em $D$ tal que

$$
\forall Y \in D,\left.i_{U} \mathrm{~d} \omega\right|_{D}(Y)=\mathrm{d} \omega(Z, Y)
$$

ou seja,

$$
\forall Y \in D, \mathrm{~d} \omega(U, Y)=\mathrm{d} \omega(Z, Y)
$$

Seja $\xi=U-Z$,

$$
\omega(\xi)=\omega(U)-\omega(Z)=1 .
$$

Se $W$ é um campo em $M$, podemos escrevê-lo

$$
\begin{aligned}
W & =\alpha \xi+Y \\
i_{\xi} \mathrm{d} \omega(W) & =\mathrm{d} \omega(\xi, W)=\mathrm{d} \omega(\xi, \alpha \xi+Y) \\
& =\mathrm{d} \omega(\xi, \alpha \xi)+\mathrm{d} \omega(\xi, Y)=0+\mathrm{d} \omega(\xi, Y)=\mathrm{d} \omega(U-Z, Y) \\
& =\mathrm{d} \omega(U, Y)-\mathrm{d} \omega(Z, Y)=0,
\end{aligned}
$$

o que nos mostra a existência de tal campo.

Vamos mostrar a unicidade.

Seja $\xi_{1}$ um campo com as propriedades da proposição. Então,

$$
\begin{aligned}
& i_{\xi_{1}} \mathrm{~d} \omega=i_{\xi} \mathrm{d} \omega=0 \quad \text { e } \quad \omega\left(\xi_{1}\right)=\omega(\xi)=1, \\
& \omega\left(\xi_{1}-\xi\right)=0 \rightarrow \xi_{1}-\xi \in D, \\
& \forall X \in D, \quad \mathrm{~d} \omega(\xi, X)=\mathrm{d} \omega\left(\xi_{1}, X\right)=0, \\
& \forall X \in D, \quad \mathrm{~d} \omega\left(\xi_{1}-\xi, X\right)=0 .
\end{aligned}
$$

Como d $\omega$ é não degenerada em $D$, temos $\xi_{1}-\xi=0$, o que mostra a unicidade.

No teorema que segue definiremos uma derivada covariante que será associada à variedade sub-riemanniana de contato. 
Teorema 1.1 Existe uma única derivada covariante

$$
\nabla: T M \times T M \rightarrow T M
$$

que satisfaz as propriedades

i) $\forall X \in D, \forall U \in T M, \nabla_{U} X \in D$;

ii) $\forall U \in T M, \nabla_{U} \xi=0$;

iii) $\forall U \in T M, \forall X, Y \in D, U\langle X, Y\rangle=\left\langle\nabla_{U} X, Y\right\rangle+\left\langle X, \nabla_{U} Y\right\rangle$;

iv) se $T_{\text {or }}$ é o tensor de torção de $\nabla$, então $\forall X, Y \in D$,

$T_{o r}(X, Y)=\mathrm{d} \omega(X, Y) \xi e$

$T_{\text {or }}(\xi, X)=\tau(X)$, onde $\tau(X)$ é um tensor simétrico $e$

$i_{\xi} \tau=0$.

Na demonstração deste teorema explicitaremos os coeficientes da derivada covariante para um referencial ortonormal local em $M$ assim determinando a existência e unicidade da derivada covariante para abertos de $M$. Para uma cobertura por abertos de $M$ teremos a derivada covariante definida para cada aberto. Essa derivada covariante será independente do referencial adotado pois para dois referenciais sobre os abertos $U_{1}$ e $U_{2}$ teremos duas derivadas covariantes $\nabla_{1}$ e $\nabla_{2}$ únicas em $U_{1}$ e $U_{2}$. Na intersecção destes abertos a existência de uma derivada covariante já está garantida por $\nabla_{1}$ e $\nabla_{2}$. Como se ela existir será única, então $\nabla_{1}$ e $\nabla_{2}$ coincidem em $U_{1} \cap U_{2}$. Tomando então uma cobertura por abertos de $M$ garantimos a existência e unicidade de $\nabla$.

Demonstração: Seja $\left\{e_{i}, \xi\right\}$ um referencial local ortonormal sobre $M$. Coloquemos

$$
\begin{array}{r}
{\left[e_{i}, e_{j}\right]=\sum_{k} c_{i j}^{k} e_{k}+a_{i j} \xi \quad \text { e } \quad\left[\xi, e_{j}\right]=\sum_{k} c_{j}^{k} e_{k}+c_{j} \xi} \\
\mathrm{d} \omega\left(e_{i}, e_{j}\right)=e_{i}\left(\omega\left(e_{j}\right)\right)-e_{j}\left(\omega\left(e_{i}\right)\right)-\omega\left(\left[e_{i}, e_{j}\right]\right)=-\omega\left(\left[e_{i}, e_{j}\right]\right)
\end{array}
$$




$$
\begin{aligned}
& =-\omega\left(\sum_{k} c_{i j}^{k} e_{k}+a_{i j} \xi\right)=-\sum_{k} c_{i j}^{k} \omega\left(e_{k}\right)-a_{i j} \omega(\xi) \\
& =-a_{i j} \\
\mathrm{~d} \omega\left(\xi, e_{j}\right) & =\xi\left(\omega\left(e_{j}\right)\right)-e_{j}(\omega(\xi))-\omega\left(\left[\xi, e_{j}\right]\right) \\
& =\xi(0)-e_{j}(1)-\omega\left(\left[\xi, e_{j}\right]\right)=-\omega\left(\left[\xi, e_{j}\right]\right) \\
& =-\omega\left(\sum_{k} c_{j}^{k} e_{k}+c_{j} \xi\right)=-\sum_{k} c_{j}^{k} \omega\left(e_{k}\right)-c_{j} \omega(\xi)=-c_{j} .
\end{aligned}
$$

Como $i_{\xi} \mathrm{d} \omega\left(e_{j}\right)=0$, temos $\mathrm{d} \omega\left(\xi, e_{j}\right)=0$. Logo, $c_{j}=0$. Se $\nabla$ satisfaz a condição $i$, temos

$$
\nabla_{e_{i}} e_{j}=\sum_{k} \Gamma_{i j}^{k} e_{k} \quad \text { e } \quad \nabla_{\xi} e_{j}=\sum_{k} \Gamma_{j}^{k} e_{k}
$$

Assim,

$$
\begin{aligned}
T_{o r}\left(e_{i}, e_{j}\right) & =\nabla_{e_{i}} e_{j}-\nabla_{e_{j}} e_{i}-\left[e_{i}, e_{j}\right] \\
& =\sum_{k} \Gamma_{i j}^{k} e_{k}-\sum_{k} \Gamma_{j i}^{k} e_{k}-\sum_{k} c_{i j}^{k} e_{k}-a_{i j} \xi \\
& =\sum_{k}\left(\Gamma_{i j}^{k}-\Gamma_{j i}^{k}-c_{i j}^{k}\right) e_{k}-a_{i j} \xi
\end{aligned}
$$

Se $\nabla$ satisfaz a condição iv, então

$$
T_{o r}\left(e_{i}, e_{j}\right)=\mathrm{d} \omega\left(e_{i}, e_{j}\right) \xi
$$

e, portanto,

$$
\Gamma_{i j}^{k}-\Gamma_{j i}^{k}-c_{i j}^{k}=0
$$

Se $\nabla$ satisfaz a condição iii, então

$$
0=e_{i}\left\langle e_{j}, e_{k}\right\rangle=\left\langle\nabla_{e_{i}} e_{j}, e_{k}\right\rangle+\left\langle e_{j}, \nabla_{e_{i}} e_{k}\right\rangle=\Gamma_{i j}^{k}+\Gamma_{k i}^{j}
$$

ou seja,

$$
\Gamma_{i j}^{k}=-\Gamma_{i k}^{j} .
$$

Permutando os índices em (I), temos

$$
\Gamma_{i j}^{k}-\Gamma_{j i}^{k}=c_{i j}^{k}
$$




$$
\begin{aligned}
\Gamma_{j k}^{i}-\Gamma_{k j}^{i} & =c_{j k}^{i} \\
\Gamma_{k i}^{j}-\Gamma_{i k}^{j} & =c_{k i}^{j} .
\end{aligned}
$$

Somando a primeira e a terceira igualdades e subtraindo a segunda, obtemos

$$
\Gamma_{i j}^{k}-\Gamma_{i k}^{j}-\Gamma_{j i}^{k}-\Gamma_{j k}^{i}+\Gamma_{k j}^{i}+\Gamma_{k i}^{j}=c_{i j}^{k}-c_{j k}^{i}+c_{k i}^{j}
$$

Utilizando (II), temos

$$
\begin{aligned}
2 \Gamma_{i j}^{k} & =c_{i j}^{k}-c_{j k}^{i}+c_{k i}^{j} \\
\Gamma_{i j}^{k} & =\frac{1}{2}\left(c_{i j}^{k}-c_{j k}^{i}+c_{k i}^{j}\right) \\
T_{o r}\left(\xi, e_{j}\right) & =\nabla_{\xi} e_{j}-\nabla_{e_{j}} \xi-\left[\xi, e_{j}\right], \text { pela propriedade ii, } \\
T_{o r}\left(\xi, e_{j}\right) & =\nabla_{\xi} e_{j}-\left[\xi, e_{j}\right]=\sum_{k} \Gamma_{j}^{k} e_{k}-\sum_{k} c_{j}^{k} e_{k} \\
& =\sum_{k}\left(\Gamma_{j}^{k}-c_{j}^{k}\right) e_{k} .
\end{aligned}
$$

Se $\nabla$ satisfaz iv, temos

$$
T_{o r}\left(\xi, e_{j}\right)=\sum_{k} \tau^{k}\left(e_{j}\right) e_{k} \quad \text { e } \quad \tau^{k}\left(e_{j}\right)=\Gamma_{j}^{k}-c_{j}^{k}
$$

Sendo o tensor $\tau$ simétrico, temos

$$
\begin{aligned}
\left\langle\tau\left(e_{i}\right), e_{j}\right\rangle=\left\langle e_{i}, \tau\left(e_{j}\right)\right\rangle & \rightarrow\left\langle\sum_{k} \tau^{k}\left(e_{i}\right) e_{k}, e_{j}\right\rangle=\left\langle e_{i}, \sum_{k} \tau^{k}\left(e_{j}\right) e_{k}\right\rangle \\
\rightarrow & \tau^{j}\left(e_{i}\right)=\tau^{i}\left(e_{j}\right) .
\end{aligned}
$$

De iii, temos

$$
0=\xi\left\langle e_{i}, e_{j}\right\rangle=\left\langle\nabla_{\xi} e_{i}, e_{j}\right\rangle+\left\langle e_{i}, \nabla_{\xi} e_{j}\right\rangle=\left\langle\sum_{k} \Gamma_{i}^{k} e_{k}, e_{j}\right\rangle+\left\langle e_{i}, \sum_{k} \Gamma_{j}^{k} e_{k}\right\rangle=\Gamma_{i}^{j}+\Gamma_{j}^{i} .
$$

Permutando os índices em (III), temos

$$
\begin{aligned}
\tau^{k}\left(e_{j}\right) & =\Gamma_{j}^{k}-c_{j}^{k}, \\
\tau^{j}\left(e_{k}\right) & =\Gamma_{k}^{j}-c_{k}^{j} .
\end{aligned}
$$


Subtraindo as duas igualdades, vem

$$
\begin{aligned}
0 & =\Gamma_{j}^{k}-\Gamma_{k}^{j}-c_{j}^{k}+c_{k}^{j} \rightarrow 2 \Gamma_{j}^{k}=c_{j}^{k}-c_{k}^{j}, \\
\Gamma_{j}^{k} & =\frac{1}{2}\left(c_{j}^{k}-c_{k}^{j}\right) .
\end{aligned}
$$

Assim, determinamos os coeficientes $\Gamma_{i j}^{k}$ e $\Gamma_{j}^{k}$ para a derivada covariante garantindo a sua existência e unicidade.

Da demonstração do Teorema 1, podemos observar que o tensor simétrico $\tau$ tem a forma $\tau_{j}^{k}=-\left(c_{j}^{k}+c_{k}^{j}\right) / 2$, pois

$$
\begin{aligned}
\tau_{j}^{k} & =\tau^{k}\left(e_{j}\right)=\Gamma_{j}^{k}-c_{j}^{k}, \\
\tau_{k}^{j} & =\tau^{j}\left(e_{k}\right)=\Gamma_{k}^{j}-c_{k}^{j} .
\end{aligned}
$$

Somando as duas igualdades e utilizando $\tau_{j}^{k}=\tau_{k}^{j}$ e $\Gamma_{j}^{k}+\Gamma_{k}^{j}=0$, temos $2 \tau_{j}^{k}=-c_{j}^{k}-c_{k}^{j}$. Logo, $\tau_{j}^{k}=-\left(c_{j}^{k}+c_{k}^{j}\right) / 2$.

Proposição 1.6 O tensor de torção pode ser escrito na forma

$$
T_{o r}=\omega \wedge \tau+\mathrm{d} \omega \otimes \xi .
$$

Seja $L^{*}=u^{1} \xi+X, V=v^{1} \xi+Y$,

$$
\begin{aligned}
& T_{\text {or }}(U, V)= T_{\text {or }}\left(u^{1}{ }^{1} \xi+X, v^{1} \xi+Y\right)=T_{o r}\left(u^{1} \xi, v^{1} \xi\right)+T_{o r}\left(u^{1} \xi, Y\right) \\
&+T_{o r}\left(X, v^{1} \xi\right)+T_{o r}(X, Y)=u \cdot v^{1} T_{o r}(\xi, \xi)+u^{1} T(\xi, Y) \\
&+v^{1} T_{o r}(X, \xi)+T_{o r}(X, Y)=u^{1} \tau(Y)-v^{1} \tau(X)+\mathrm{d} \omega(X, Y) \xi, \\
&(\omega \wedge \tau+\mathrm{d} \omega \otimes \xi)(U, V)=(\omega \wedge \tau)(U, V)+\mathrm{d} \omega(U, V) \xi \\
&=\omega\left(u^{1} \xi+X\right) \tau\left(v^{1} \xi+Y\right)-\omega\left(v^{1} \xi+Y\right) \tau\left(u^{1} \xi+X\right)+\mathrm{d} \omega\left(u^{1} \xi+X, v^{1} \xi+Y\right) \xi \\
&=\left(u^{1} \cdot \omega(\xi)+\omega(X)\right) \tau\left(v^{1} \xi+Y\right)-\left(v^{1} \omega(\xi)+\omega(Y)\right) \tau\left(u^{1} \xi+X\right)
\end{aligned}
$$




$$
\begin{aligned}
& +u^{1} v^{1} \mathrm{~d} \omega(\xi, \xi) \xi+v^{1} \mathrm{~d} \omega(X, \xi) \xi+u^{1} \mathrm{~d} \omega(\xi, Y) \xi+\mathrm{d} \omega(X, Y) \xi \\
= & u{ }^{1} \tau\left(v^{1} \xi+Y\right)-v^{1} \tau\left(u^{1} \xi+X\right)+\mathrm{d} \omega(X, Y) \xi \\
= & u^{1} \tau(Y)-v^{1} \tau(X)+\mathrm{d} \omega(X, Y) \xi+u^{1} v^{1} \tau(\xi)-v^{1} u^{1} \tau(\xi) \\
= & u \cdot{ }^{1} \tau(Y)-v^{1} \tau(X)+\mathrm{d} \omega(X, Y) \xi .
\end{aligned}
$$

Assim, $T_{o r}(U, V)=(\omega \wedge \tau+\mathrm{d} \omega \otimes \xi)(U, V)$.

Lembrando a definição da derivada covariante de uma 1-forma,

$$
\nabla_{U} \eta(V)=U(\eta(V))-\eta\left(\nabla_{U} V\right)
$$

temos as seguintes proposições.

Proposição 1.7 Para a forma de contato da variedade sub-riemanniana vale a relação $\nabla \omega=0$.

De fato,

$$
\begin{aligned}
\nabla_{U} \omega(V) & =U(\omega(V))-\omega\left(\nabla_{U} V\right) \\
\nabla_{U} \omega\left(v^{1} \xi+Y\right) & =U\left(\omega\left(v^{1} \xi+Y\right)\right)-\omega\left(\nabla_{U}\left(v^{1} \xi+Y\right)\right) \\
& =U\left(v^{1} \omega(\xi)+\omega(Y)\right)-\omega\left(\nabla_{U}\left(v^{1} \xi\right)+\nabla_{U} Y\right) \\
& =U\left(v^{1}\right)-\omega\left(\nabla_{U}\left(v^{1} \xi\right)\right)-\omega\left(\nabla_{U} Y\right) \\
& =U\left(v^{1}\right)-\omega\left(U\left(v^{1}\right) \xi+v^{1} \nabla_{U} \xi\right)=U\left(v^{1}\right)-\omega\left(U\left(v^{1}\right) \xi\right) \\
& =U\left(v^{1}\right)-U\left(v^{1}\right)=0 .
\end{aligned}
$$

Vamos utilizar a seguinte notação:

$$
\widehat{\nabla} \eta(U, V)=\left(\nabla_{U} \eta\right)(V)-\left(\nabla_{V} \eta\right)(U)
$$

Proposição 1.8 Sendo $\eta$ uma 1-forma, vale a relação

$$
\mathrm{d} \eta=\widehat{\nabla} \eta+\eta \circ T_{o r}
$$


De fato,

$$
\begin{aligned}
\mathrm{d} \eta\left(C^{:} V\right) & =U(\eta(V))-V(\eta(U))-\eta([U, V]) \\
\widehat{\nabla} \eta(U: V) & =U(\eta(V))-\eta\left(\nabla_{U} V\right)-V(\eta(U))+\eta\left(\nabla_{V} U\right) \\
& =U(\eta(V))-V(\eta(U))-\eta\left(\nabla_{U} V-\nabla_{V} U\right) \\
& =U(\eta(V))-V(\eta(U))-\eta\left(T_{o r}(U V)+[U, V]\right) \\
& =U(\eta(V))-V(\eta(U))-\eta([U, V])-\eta\left(T_{o r}(U, V)\right) \\
& =\mathrm{d} \eta(U, V)-\eta(T(U, V)) .
\end{aligned}
$$

Assim,

$$
\mathrm{d} \eta(U, V)=\widehat{\nabla} \eta(U, V)+\eta\left(T_{o r}(U, V)\right) .
$$

Em particular, para a forma de contato temos

$$
\mathrm{d} \omega=\widehat{\nabla} \omega+\omega \circ T_{\text {or }}
$$

Seja $\left\{e_{i}\right\}, 1 \leq i \leq 2 n+1$, uma base local ortonormal de campos e seja $\left\{\theta^{i}\right\}, 1 \leq i \leq$ $2 n+1$, sua base dual.

Podemos escrever a derivada covariante destes campos na forma $\nabla e_{i}=\sum_{j} \omega_{i}^{j} e_{j}$, onde $\omega_{i}^{j}$ são 1-formas.

As formas $\omega_{i}^{j}$ são chamadas de formas de conexão.

Proposição 1.9 Sendo $\nabla$ compativel com a métrica, vale a relação $\omega_{i}^{j}=-\omega_{j}^{i}$ para as formas de conexão.

De fato,

$$
\begin{aligned}
0 & =\nabla\left\langle e_{i}, e_{j}\right\rangle=\left\langle\nabla e_{i}, e_{j}\right\rangle+\left\langle e_{i}, \nabla e_{j}\right\rangle \\
& =\left\langle\sum_{k} \omega_{i}^{k} e_{k}, e_{j}\right\rangle+\left\langle e_{i}, \sum_{k} \omega_{j}^{k} e_{k}\right\rangle=\omega_{i}^{j}+\omega_{j}^{i} .
\end{aligned}
$$


Proposição 1.10 Valem as igualdades

$$
\mathrm{d} \theta^{i}=-\sum_{j} \omega_{j}^{i} \wedge \theta^{j}+T_{o r}^{i}
$$

para $1 \leq i \leq 2 n+1$

De fato,

$$
\nabla_{U} \theta^{i}\left(e_{j}\right)=U\left(\theta^{i}\left(e_{j}\right)\right)-\theta^{i}\left(\nabla_{U} e_{j}\right)=U \delta_{i j}-\theta^{i}\left(\nabla_{U} e_{j}\right)=-\theta^{i}\left(\nabla_{U}\right)
$$

Assim,

$$
\begin{aligned}
\nabla \theta^{i}\left(e_{j}\right) & =-\theta^{i}\left(\nabla e_{j}\right)=-\theta^{i}\left(\sum_{k} \omega_{j}^{k} e_{k}\right)=-\omega_{j}^{i} \\
\nabla \theta^{i}\left(e_{j}\right) & =-\omega_{j}^{i} \rightarrow \nabla \theta^{i}=-\sum_{j} \omega_{j}^{i} \otimes \theta^{j} \\
\widehat{\nabla} \theta^{i}(U, V) & =\left(\nabla_{U} \theta^{i}\right)(V)-\left(\nabla_{V} \theta^{i}\right)(U) \\
& =-\sum_{j} \omega_{j}^{i} \otimes \theta^{j}(U, V)+\sum_{j} \omega_{j}^{i} \otimes \theta^{j}(U, V)=-\sum_{j}\left(\omega_{j}^{i} \wedge \theta^{j}\right)(U, V) .
\end{aligned}
$$

Logo,

$$
\widehat{\nabla} \theta^{i}=-\sum_{j} \omega_{j}^{i} \wedge \theta^{j}
$$

Pela Proposição 1.8, temos

$$
\mathrm{d} \theta^{i}=\widehat{\nabla} \theta^{i}+\theta^{i} \circ T_{o r}=-\sum_{j} \omega_{j}^{i} \wedge \theta^{j}+T_{o r}^{i} .
$$

A equação $\mathrm{d} \theta^{i}=-\sum_{j} \omega_{j}^{i} \wedge \theta^{i}+T^{i}$ é chamada de primeira equação de estrutura.

Vamos escrever a primeira equação de estrutura para a derivada covariante da geometria sub-riemanniana.

Seja $\left\{e_{i}\right\}, 1 \leq i \leq 2 n$, uma base local de $D$ ortonormal e $\left\{e_{i}, \xi\right\}$ base local de $M$ com base dual $\left\{\theta^{i}, \omega\right\}$.

Pelas propriedades i e ii da conexão, temos

$$
\nabla e_{i}=\sum_{j} \omega_{i}^{j} e_{j} \quad \text { e } \quad \nabla \xi=0
$$




$$
\begin{aligned}
\widehat{\nabla} \theta^{i} & =-\sum_{j} \omega_{j}^{i} \wedge \theta^{j} \quad \text { e } \quad \widehat{\nabla} \omega=0 \\
\mathrm{~d} \theta^{i} & =-\sum_{j} \omega_{j}^{i} \wedge \theta^{j}+\theta^{i} \circ T_{o r} \\
\mathrm{~d} \omega & =\widehat{\nabla} \omega+\omega \circ T=\omega \circ T_{o r}
\end{aligned}
$$

Como $T_{\text {or }}=\omega \wedge \tau+\mathrm{d} \omega \otimes \xi$, temos

$$
\theta^{i} \circ T_{o r}=\theta^{i}(\omega \wedge \tau+\mathrm{d} \omega \otimes \xi)=\theta^{i}(\omega \wedge \tau)
$$

Sendo $\tau=\sum_{j} \tau^{j} \otimes e_{j}$, temos

$$
\begin{aligned}
\theta^{i} \circ T_{o r} & =\theta^{i}\left(\omega \wedge \sum_{j} \tau^{j} \otimes e_{j}\right)=\omega \wedge \tau^{i} \\
\omega \circ T_{o r} & =\omega(\omega \wedge \tau+\mathrm{d} \omega \otimes \xi)=\omega(\omega \wedge \tau)+\omega(\mathrm{d} \omega \otimes \xi)=\mathrm{d} \omega .
\end{aligned}
$$

Assim, a primeira equação de estrutura para a derivada covariante da geometria subriemanniana é escrita

$$
\begin{aligned}
& \mathrm{d} \theta^{i}+\sum_{j} \omega_{j}^{i} \wedge \theta^{j}=\omega \wedge \tau^{i}, \\
& \mathrm{~d} \omega=\mathrm{d} \omega .
\end{aligned}
$$

Proposição 1.11 Sendo $X$ um campo tangente à distribuição $D$, vale a relação $[\xi, X] \in$ D.

De fato,

$$
\begin{aligned}
\mathrm{d} \omega(\xi, X) & =\xi \omega(X)-X \omega(\xi)-\omega[\xi, X]=\xi .0-X .1-\omega[\xi, X] \\
\mathrm{d} \omega(\xi, X) & =i_{\xi} \mathrm{d} \omega(X)=0 .
\end{aligned}
$$

Logo,

$$
\omega[\xi, X]=0
$$

Assim, $[\xi, X] \in D$. 


\section{Capítulo 2}

\section{Variação da energia}

Serão consideradas variedades conexas. Todas as curvas são contínuas.

Seja $(M, D, g)$ uma variedade sub-riemanniana de contato.

Definição 2.1 Uma curva $c:[a, b] \rightarrow M$, suave por partes na partição $P=\{a=$ $\left.t_{0}, \ldots, t_{l}=b\right\}$ é chamada tangente a $D$ se

$$
\begin{aligned}
& \forall t \in[a, b]-P, \quad \dot{c}(t) \in D_{c(t)} \\
& \forall t \in P-\{a, b\}, \quad \dot{c}\left(t^{+}\right), \dot{c}\left(t^{-}\right) \in D_{c(t)} \\
& \dot{c}\left(a^{+}\right) \in D_{c(a)}, \quad \dot{c}\left(b^{-}\right) \in D_{c(b)},
\end{aligned}
$$

onde

$$
\dot{c}\left(t_{i}^{+}\right)=\lim _{t \rightarrow t_{i}^{+}} \dot{c}(t) \quad \text { e } \quad \dot{c}\left(t_{i}^{-}\right)=\lim _{t \rightarrow t_{i}^{-}} \dot{c}\left(t_{i}\right) .
$$

O conjunto das curvas suaves por partes definidas em $[a, b]$ e tangentes a $D$ será denotada por $\Omega(D)$.

Utilizaremos a notação $\dot{c} \in D$ para indicar que $c$ é tangente a $D$.

Assim, $\Omega(D)=\{c:[a, b] \rightarrow M \mid \dot{c} \in D\}$.

Sejam $p, q \in M$. Denotaremos por $\Omega_{p q}(D)$ o subconjunto de $\Omega(D)$

$$
\begin{aligned}
\Omega_{p q}(D) & =\{c:[a, b] \rightarrow M \mid \dot{c} \in D, c(a)=p, c(b)=q\} \\
& =\{c \in \Omega(D) \mid c(a)=p, c(b)=q\}
\end{aligned}
$$


e por $\Omega_{p}(D)$ o subconjunto

$$
\Omega_{p}(D)=\{c \in \Omega(D) \mid c(a)=p\}
$$

$\operatorname{Em} \Omega(D)$ definimos o funcional comprimento de arco $L: \Omega(D) \rightarrow \mathbb{R}$ por

$$
L(c)=\int_{a}^{b}\langle\dot{c}, \dot{c}\rangle^{1 / 2} \mathrm{~d} t
$$

onde $\langle X, Y\rangle=g(X, Y)$.

Teorema 2.1 (Chow) $\forall p, q \in M, \Omega_{p q}(D) \neq \emptyset$.

Demonstração: De fato, pelo teorema de Darboux (ref. Godbillon), $\forall p \in M \exists\left(V_{p}, \varphi_{p}\right)$, $\varphi_{p}=\left(x_{1}, \ldots, x_{2 n}, z\right), \varphi_{p}(p)=0$, sistema de coordenadas onde a forma de contato $\omega$ é escrita

$$
\omega=\mathrm{d} z-\sum_{i=1}^{n} x_{i} \mathrm{~d} x_{i+n}
$$

Tomemos $U_{p}^{1} \subset \varphi_{p}\left(V_{p}\right)$ da forma $U_{p}^{1}=\left[\prod_{1}^{2 n}(-a, a)\right] \times\left(-a^{2}, a^{2}\right)$.

Seja $V_{p}^{1}=\varphi_{p}^{-1}\left(U_{p}^{1}\right)$. Vamos mostrar que $\forall q \in V_{p}^{1}$ podemos construir uma curva tangente à distribuição com extremos em $p$ e $q$ e cuja imagem está contida em $V_{p}^{1}$.

Seja $\varphi_{p}(q)=\left(b_{1}, \ldots, b_{2 n+1}\right)$.

O segmento de reta

$$
\begin{aligned}
r_{1}:[0,1] & \rightarrow U_{p}^{1} \\
t & \rightarrow\left(b_{1} t, \ldots, b_{n} t, b_{n+1}, \ldots, b_{2 n+1}\right)
\end{aligned}
$$

tem extremos em $\left(b_{1}, \ldots, b_{2 n+1}\right)$ e $\left(0, \ldots, 0, b_{n+1}, \ldots, b_{2 n+1}\right)$ e é tangente à distribuição pois

$$
\omega\left(\dot{r}_{1}\right)=\left(\mathrm{d} z-\sum_{i=1}^{n} x_{i} \mathrm{~d} x_{i+n}\right)\left(b_{1}, \ldots, b_{n}, 0, \ldots, 0\right)=0 .
$$

O segmento de reta

$$
\begin{aligned}
r_{2}:[0,1] & \rightarrow U_{p}^{1} \\
t & \rightarrow\left(0, \ldots, 0, b_{n+1} t, \ldots, b_{2 n} t, b_{2 n+1}\right)
\end{aligned}
$$


tem extremos em $\left(0, \ldots, 0, b_{n+1}, \ldots, b_{2 n+1}\right)$ e $\left(0, \ldots, 0, b_{2 n+1}\right)$ e é tangente à distribuição pois

$$
\omega\left(\dot{r}_{2}\right)=\left(\mathrm{d} z-\sum_{i=1}^{n} x_{i} \mathrm{~d} x_{i+n}\right)\left(0, \ldots, 0, b_{n+1}, \ldots, b_{2 n}, 0\right)=0 .
$$

Vamos assumir o caso em que $b_{2 n+1}>0$. O segmento de reta

$$
\begin{aligned}
r_{3}:[0,1] & \rightarrow U_{p}^{1} \\
t & \rightarrow\left(0, \ldots, 0, \sqrt{b_{2 n+1}} t, 0, \ldots, 0, b_{2 n+1}\right)=\sqrt{b_{2 n+1}} t e_{n}+b_{2 n+1} e_{2 n+1}
\end{aligned}
$$

tem extremos em $\left(0, \ldots, b_{2 n+1}\right)$ e $\left(0, \ldots, 0, \sqrt{b_{2 n+1}}, 0, \ldots, b_{2 n+1}\right)$ e é tangente à distribuição pois

$$
\omega\left(\dot{r}_{3}\right)=\omega\left(0, \ldots, 0, \sqrt{b_{2 n+1}}, 0, \ldots, 0\right)=0
$$

O segmento de reta

$$
\begin{aligned}
r_{4}:[0,1] & \rightarrow U_{p}^{1} \\
t & \rightarrow\left(\sqrt{b_{2 n+1}} t, 0, \ldots, 0, \sqrt{b_{2 n+1}}, 0, \ldots, b_{2 n+1}\right)
\end{aligned}
$$

tem extremos em $\left(0, \ldots, 0, \sqrt{b_{2 n+1}}, 0, \ldots, 0, b_{2 n+1}\right)$ e $\left(\sqrt{b_{2 n+1}}, 0, \ldots, 0, \sqrt{b_{2 n+1}}, 0, \ldots, b_{2 n+1}\right)$ e é tangente à distribuição pois

$$
\omega\left(\dot{r}_{4}\right)=\omega\left(\sqrt{b_{2 n+1}}, 0, \ldots, 0\right)=0
$$

O segmento de reta

$$
\begin{aligned}
r_{5}:[0,1] & \rightarrow U_{p}^{1} \\
t & \rightarrow\left(\sqrt{b_{2 n+1}}, 0, \ldots, 0, \sqrt{b_{2 n+1}}(1-t), 0, \ldots, 0, b_{2 n+1}(1-t)\right)
\end{aligned}
$$

tem extremos em $\left(\sqrt{b_{2 n+1}}, 0, \ldots, 0, \sqrt{b_{2 n+1}}, 0, \ldots, 0, b_{2 n+1}\right)$ e $\left(\sqrt{b_{2 n+1}}, 0, \ldots, 0\right)$ e é tangente à distribuição pois

$$
\begin{aligned}
\omega\left(\dot{r}_{5}\right) & =\left(\mathrm{d} z-\sum_{i=1}^{n} x_{i} \mathrm{~d} x_{i+n}\right)\left(0, \ldots, 0,-\sqrt{b_{2 n+1}}, 0, \ldots, 0,-b_{2 n+1}\right) \\
& =-b_{2 n+1}-\left(\sqrt{b_{2 n+1}}\right)\left(-\sqrt{b_{2 n+1}}\right)=0 .
\end{aligned}
$$


O segmento de reta

$$
\begin{aligned}
r_{6}:[0,1] & \rightarrow U_{p}^{1} \\
t & \rightarrow\left(\sqrt{b_{2 n+1}}(1-t), 0, \ldots, 0\right)
\end{aligned}
$$

tem extremos em $\left(\sqrt{b_{2 n+1}}, 0, \ldots, 0\right)$ e 0 e é tangente à distribuição pois

$$
\omega\left(\dot{r}_{6}\right)=\omega\left(-\sqrt{b_{2 n+1}}, 0, \ldots, 0\right)=0 .
$$

Assim, temos uma curva suave por partes tangente à distribuição obtida pela imagem inversa através de $\hat{\Upsilon}_{p}$ da curva suave por partes formada pelos segmentos de reta $r_{1}$ a $r_{6}$. Esta curva tem extremos em $p$ e $q$ e sua imagem está contida em $V_{p}^{1}$.

O caso $b_{2 n+1}<0$ é análogo e se $b_{2 n+1}=0$ bastará utilizarmos os segmentos de reta $r_{1}$ e $r_{2}$.

Seja $N=\left\{q \in M \mid \exists c:[a, b] \rightarrow M, c \in \Omega_{p}(D), c(b)=q\right\}$.

Seja $r \in N$. Como vimos anteriormente, existe um aberto $V_{r}^{1}$ para o qual cada ponto pode ser ligado a $r$ por uma curva suave por partes tangente à distribuição. Assim, estes pontos também podem ser ligados a $p$, o que mostra que $V_{r}^{1} \subset N$ e, portanto, $N$ é aberto.

Se $r \notin N$, então nenhum ponto de $V_{r}^{1}$ pode pertencer a $N$, caso contrário, poderíamos ligar $r$ a $p$ por uma curva suave por partes tangente à distribuição, o que mostra que $N$ é fechado.

Como $M$ é conexa, temos $N=M$, o que termina a demonstração.

Utilizando o funcional comprimento de arco definimos a distância de dois pontos $p$ e $q$ de $M$ por

$$
d(p, q)=\inf \left\{L(c) \mid c \in \Omega_{p q}(D)\right\}
$$

Mostremos que $d$ é uma distância. Para tanto, seja $g_{1}$ uma métrica que estende $g$ de forma que $g_{1}(\xi, \xi)=1$ e $\xi \perp D$. 
Sendo $c \in \Omega_{p q}(D)$, temos

$$
L(c)=\int_{a}^{b} g(\dot{c}, \dot{c})^{1 / 2} \mathrm{~d} t=\int_{a}^{b} g_{1}(\dot{c}, \dot{c})^{1 / 2} \mathrm{~d} t=L_{1}(c) .
$$

Sejam $p, q \in M, p \neq q$, e $\Omega_{p q}=\{c:[a, b] \rightarrow M \mid c(a)=p, c(b)=q\}$.

$d_{1}(p, q)=\inf \left\{L_{1}(c) \mid c \in \Omega_{p q}\right\}$ é a distância associada a $g_{1}$.

Como $\Omega_{p q}(D) \subset \Omega_{p q}$ e $\left.L_{1}\right|_{\Omega_{p q}(D)}=L$, temos

$$
0<d_{1}(p, q)=\inf \left\{L_{1}(c) \mid c \in \Omega_{p q}\right\} \leq \inf \left\{L_{1}(c) \mid c \in \Omega_{p q}(D)\right\}=d(p, q) .
$$

Sejam $p, q, r \in M$. Como $\Omega_{p r}(D)$ contém o conjunto das curvas suaves por partes que têm o ponto $q$ em sua imagem, temos

$$
d(p, r) \leq d(p, q)+d(q, r) .
$$

Finalmente, $d(p, q)=d(q, p)$, já que o comprimento de arco independe da parametrização da curva.

Definição 2.2 Uma curva $c \in \Omega_{p q}(D)$ é chamada minimizante se

$$
\forall \gamma \in \Omega_{p q}(D), \quad L(c) \leq L(\gamma)
$$

Caso exista uma curva minimizante, temos $d(p, q)=L(c)$ e a curva minimizante é um extremo do funcional $L$.

Estamos interessados em conhecer as curvas extremais do funcional $L$. Isto pode ser feito com o auxílio do funcional energia definido por

$$
\begin{aligned}
& E: \Omega_{p q}(D) \rightarrow \mathbb{R} \\
& E(c)=\frac{1}{2} \int_{a}^{b}\langle\dot{c}, \dot{c}\rangle \mathrm{d} t
\end{aligned}
$$

Da desigualdade de Cauchy-Schwarz temos

$$
\left(\int_{a}^{b} f . g \mathrm{~d} t\right)^{2} \leq \int_{a}^{b} f^{2} \mathrm{~d} t . \int_{a}^{b} g^{2} \mathrm{~d} t
$$


Tomemos $g$ a função constante igual a 1 . A igualdade vale se e somente se $f$ for constante. Aplicando ao caso $f=\|\dot{c}\|$, temos

$$
L(c)^{2} \leq 2 E(c)(b-a)
$$

e a igualdade é válida se e somente se $\|\dot{c}\|$ é constante.

Assim, se $c$ minimiza $L$ e tem velocidade constante, temos

$$
2 E(c)(b-a)=L(c)^{2} \leq L(\gamma)^{2} \leq 2 E(\gamma)(b-a) \rightarrow E(c) \leq E(\gamma)
$$

e $c$ minimiza $E$.

Sejam $M$ e $N$ variedades e $f: N \rightarrow M$.

Definição 2.3 Um campo ao longo de $f$ é uma secção $\widehat{V}: N \rightarrow T M$ tal que

$$
\widehat{V}(p) \in T_{f(p)} M
$$

Seja $c:[a, b] \rightarrow M$ uma curva suave por partes na partição $P=\left\{a=t_{0}, \ldots, t_{l}=b\right\}$.

Definição 2.4 Uma variação de c é uma função contínua

$$
\begin{aligned}
\Gamma:[a, b] \times(-\varepsilon, \varepsilon) & \rightarrow M \\
(t, s) & \rightarrow \Gamma(t, s)
\end{aligned}
$$

diferenciável em s e suave por partes em t, isto é,

$$
\forall t \in[a, b],\left.\quad \Gamma\right|_{\{t\} \times(-\varepsilon, \varepsilon)}
$$

é diferenciável em $s$ e

$$
\forall s \in[-\varepsilon, \varepsilon],\left.\quad \Gamma\right|_{[a, b] \times\{s\}}
$$

é diferenciável por partes na partição $P$.

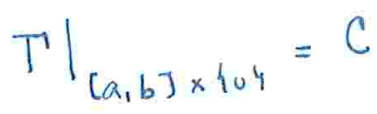


Definição 2.5 A variação infinitesimal de $\Gamma$ é o campo ao longo de c dado por $V(t)=$ $\frac{\partial \Gamma}{\partial s}(t, 0)$.

Notações: Seja $s \in(-\varepsilon, \varepsilon)$, então $c_{s}$ é a curva

$$
\begin{aligned}
& c_{s}:[a, b] \rightarrow M, \quad c_{s}(t)=\Gamma(t, s) \\
& T(t, s)=\frac{\partial \Gamma}{\partial t}(t, s), \quad T\left(t_{i}^{+}, s\right)=\lim _{t \rightarrow t_{i}^{+}} \frac{\partial \Gamma}{\partial t}(t, s), \\
& T\left(t_{i}^{-}, s\right)=\lim _{t \rightarrow t_{i}^{-}} \frac{\partial \Gamma}{\partial t}(t, s), \quad V(t, s)=\frac{\partial \Gamma}{\partial s}(t, s) .
\end{aligned}
$$

Definição 2.6 Г é uma variação de contato de c se e somente se

$$
\forall s \in(-\varepsilon, \varepsilon), \quad c_{s} \in \Omega(D)
$$

Definição $2.7 V(t)=\frac{\partial \Gamma}{\partial s}(t, 0)$ é uma variação infinitesimal de contato se e somente se $\Gamma$ é uma variação de contato.

A aplicação $\tilde{c}:(-\varepsilon, \varepsilon) \rightarrow \Omega(D) \quad$ é uma curva em $\Omega(D)$ e nos motiva as seguintes definições.

$$
s \rightarrow \tilde{c}(s)=c_{s}
$$

Definição 2.8 Um vetor tangente a $\Omega(D)$ é uma variação infinitesimal de contato.

Definição 2.9 O espaço tangente $a \Omega(D)$ em c é o conjunto das variações infinitesimais de contato de $c$.

Denotamos este espaço por $T_{c} \Omega(D)$. Deste modo, $V \in T_{c} \Omega(D)$ se e somente se $V=$ $\partial \Gamma / \partial s$, onde $\Gamma$ é variação de contato de $c$.

Definição $2.10 \Gamma$ é uma variação com extremos fixos de c se e somente se

$$
\forall s \in(-\varepsilon, \varepsilon), \quad \Gamma(a, s)=c(a) \quad e \quad \Gamma(b, s)=c(b) .
$$


Para variações de contato com extremos fixos, se $c(a)=p$ e $c(b)=q$, temos $\forall s \in(-\varepsilon, \varepsilon), c_{s} \in \Omega_{p q}(D)$

Definição $2.11 V(t)=\frac{\partial \Gamma}{\partial t}(t, 0)$ é uma variação infinitesimal de contato com extremos fixos se e somente se $\Gamma$ é uma variação de contato com extremos fixos.

Definição 2.12 O espaço tangente $a \Omega_{p q}(D)$ em c, denotado por $T_{c} \Omega_{p q}(D)$ é o conjunto das variações infinitesimais de contato com extremos fixos de c.

Utilizando as notações acima,

$$
V \in T_{c} \Omega_{p q}(D) \leftrightarrow V=\frac{\partial \Gamma}{\partial s}, \quad \forall s c_{s} \in \Omega_{p q}(D)
$$

Se $V \in T_{c} \Omega_{p q}(D)$, então $V(a)=\frac{\partial \Gamma}{\partial s}(a, 0)=\frac{d}{d s} c_{s}(0)=0$ e analogamente $V(b)=0$.

Definição 2.13 A diferencial de um funcional $E: \Omega(D) \rightarrow \mathbb{R}$ em c é a aplicação $\mathrm{d} E_{c}: T_{c} \Omega(D) \rightarrow \mathbb{R}$ dada por

$$
d E_{c}(V)=\left.\frac{\mathrm{d}}{\mathrm{d} s} E\left(c_{s}\right)\right|_{s=0}
$$

Definição 2.14 Uma curva $c \in \Omega(D)$ é um ponto crítico de um funcional $E$ se e somente se $\mathrm{d} E_{c}=0$.

Seja $c \in \Omega_{p q}(D)$ minimizante e $\Gamma$ uma variação de contato com extremos fixos e $V$ a variação infinitesimal de contato a ela associada.

$\forall s \in(-\varepsilon, \varepsilon), E(c) \leq E\left(c_{s}\right)$. Logo,

$$
\mathrm{d} E_{c}(V)=\left.\frac{\mathrm{d}}{\mathrm{d} s} E\left(c_{s}\right)\right|_{s=0}=0
$$

ou seja, se $c$ é minimizante, então $\forall V \in T_{c} \Omega_{p q}(D) \mathrm{d} E_{c}(V)=0$ e $c$ é um ponto crítico de $\left.E\right|_{\Omega_{p q}(D)}$ 
Nosso objetivo agora é estudar as variações infinitesimais de contato. Para tanto faremos uso de operadores sobre o espaço das variações infinitesimais.

No que segue, consideramos curvas $c:[a, b] \rightarrow M$ de classe $C^{\infty}$.

O espaço das variações infinitesimais de $c$ é o conjunto dos campos $C^{\infty}$ ao longo de $c$ ou, de maneira equivalente, o espaço das secções $C^{\infty}$ de $c^{*}(T M)$ que denotaremos por $C^{\infty}\left(c^{*}(T M)\right)$.

Este espaço tem estrutura de módulo sobre o espaço das funções $C^{\infty}([a, b], \mathbb{R})$, onde a multiplicação é dada por

$$
(f . V)(t)=f(t) V(t), \quad f \in C^{\infty}([a, b], \mathbb{R}), \quad V \in C^{\infty}\left(c^{*}(T M)\right)
$$

Definição 2.15 Seja $f: N \rightarrow M$. Um campo tangente ao longo de $f$ é um campo $\widehat{V}$ ao longo de $f$ tal que $\widehat{V}=\mathrm{d} f(V)$, onde $V$ é um campo em $N$.

Sejam $\widehat{V}, \widehat{T}$ campos tangentes ao longo de $f$.

Definição $2.16[\widehat{V}, \widehat{T}]=\mathrm{d} f[V, T]$.

Proposição 2.1 Sejam $f: N \rightarrow M, \widehat{V}=\mathrm{d} f(V)$ e $\widehat{T}=\mathrm{d} f(T)$ campos tangentes ao longo de $f$ e $\omega: T M \rightarrow \mathbb{R}$ uma 1-forma sobre $M$. Então vale a igualdade

$$
\mathrm{d} \omega(\widehat{V}, \widehat{T})=V \omega(\widehat{T})-T \omega(\widehat{V})-\omega[\widehat{V}, \widehat{T}] .
$$

De fato, por linearidade, basta verificar a igualdade para $\omega=g \cdot \mathrm{d} h$, onde $g, h: M \rightarrow \mathbb{R}$.

$$
\begin{aligned}
& \mathrm{d} \omega=\mathrm{d} g \wedge \mathrm{d} h \\
& \mathrm{~d} \omega(\widehat{V}, \widehat{T})=(\mathrm{d} g \wedge \mathrm{d} h)(\widehat{V}, \widehat{T})=\mathrm{d} g(\widehat{V}) \mathrm{d} h(\widehat{T})-\mathrm{d} g(\widehat{T}) \mathrm{d} h(\widehat{V}) \\
& =\mathrm{d} g(\mathrm{~d} f(V)) \mathrm{d} h(\mathrm{~d} f(T))-\mathrm{d} g(\mathrm{~d} f(T)) \mathrm{d} h(\mathrm{~d} f(V)) \\
& =(\mathrm{d} g \circ \mathrm{d} f)(V)(\mathrm{d} h \circ \mathrm{d} f)(T)-(\mathrm{d} g \circ \mathrm{d} f)(T)(\mathrm{d} h \circ \mathrm{d} f)(V) \\
& =\mathrm{d}(g \circ f)(V) \mathrm{d}(h \circ f)(T)-\mathrm{d}(g \circ f)(T) \mathrm{d}(h \circ f)(V)
\end{aligned}
$$




$$
\begin{aligned}
& V \omega(\widehat{T})=V(\omega(\mathrm{d} f(T)))=V((g \circ f) \mathrm{d} h(\mathrm{~d} f(T)))=V((g \circ f) \mathrm{d}(h \circ f)(T)) \\
& =V(g \circ f) \mathrm{d}(h \circ f)(T)+(g \circ f) V(\mathrm{~d}(h \circ f)(T)) \\
& =\mathrm{d}(g \circ f)(V) \mathrm{d}(h \circ f)(T)+(g \circ f) V(\mathrm{~d}(h \circ f)(T)) \\
& T \omega(\widehat{V})=\mathrm{d}(g \circ f)(T) \mathrm{d}(h \circ f)(V)+(g \circ f) T(\mathrm{~d}(h \circ f)(V)) \\
& V \omega(\widehat{T})-T \omega(\widehat{V})=\mathrm{d} \omega(\widehat{V}, \widehat{T})+(g \circ f)(V(\mathrm{~d}(h \circ f)(T))-T(\mathrm{~d}(h \circ f)(V))) \\
& \omega[\widehat{V}, \widehat{T}]=\omega(\mathrm{d} f[V, T])=(g \circ f) \mathrm{d} h(\mathrm{~d} f[V, T])=(g \circ f) \mathrm{d}(h \circ f)([V, T]) \\
& =(g \circ f)[V, T](h \circ f)=(g \circ f)(V(T(h \circ f))-T(V(h \circ f))) \\
& =(g \circ f)(V(\mathrm{~d}(h \circ f)(T))-T(\mathrm{~d}(h \circ f)(V))) .
\end{aligned}
$$

Logo, $V \omega(\widehat{T})-T \omega(\widehat{V})=\mathrm{d} \omega(\widehat{V}, \widehat{T})+\omega[\widehat{V}, \widehat{T}]$, o que mostra a igualdade.

A seguir enunciamos uma condição necessária para que uma variação infinitesimal seja de contato. Esta condição nos motivará a definir um operador diferencial.

Proposição 2.2 Seja $V=\frac{\partial \Gamma}{\partial s} \in T_{c} \Omega(D)$ uma variação infinitesimal de contato. Então, $i_{V} \mathrm{~d} \omega(\dot{c})+\frac{\mathrm{d}}{\mathrm{d} t} i_{V} \omega=0$.

De fato, utilizando a proposição anterior, temos

$$
\begin{aligned}
& \mathrm{d} \omega(V, T)=\frac{\partial}{\partial s} \omega(T)-\frac{\partial}{\partial t} \omega(V)-\omega[V, T] \\
& {[V, T]=\mathrm{d} \Gamma\left[\frac{\partial}{\partial s}, \frac{\partial}{\partial t}\right]=\mathrm{d} \Gamma(0)=0} \\
& V \in T_{c} \Omega(D) \rightarrow \forall t \in[a, b] \quad \forall s \in(-\varepsilon, \varepsilon) \omega\left(\dot{c}_{s}(t)\right)=0 \\
& \dot{c}_{s}(t)=T(t, s) \\
& \omega(T(t, s))=0 \rightarrow \frac{\partial}{\partial s} \omega(T(t, s))=0
\end{aligned}
$$


Assim,

$$
\mathrm{d} \omega(V, T)=-\frac{\partial}{\partial t} \omega(V)
$$

Em particular, para $s=0$, temos

$$
\mathrm{d} \omega(V(t), \dot{c}(t))+\frac{\mathrm{d}}{\mathrm{d} t} \omega(V(t))=0
$$

ou seja,

$$
i_{V} \mathrm{~d} \omega(\dot{c})+\frac{\mathrm{d}}{\mathrm{d} t} i_{V} \omega=0
$$

Definição 2.17 Seja $D_{c}$ o operador

$$
\begin{aligned}
D_{c}: C^{\infty}\left(c^{*}(T M)\right) & \rightarrow C^{\infty}([a, b], \mathbb{R}) \\
V & \rightarrow D_{c}(V)=\left(i_{V} \mathrm{~d} \omega\right)(\dot{c})+\frac{\mathrm{d}}{\mathrm{d} t} i_{V} \omega
\end{aligned}
$$

Em termos deste operador podemos enunciar a proposição anterior como

$$
V \in T_{c} \Omega(D) \rightarrow V \in \operatorname{ker} D_{c} .
$$

Proposição 2.3 O operador $D_{c}$ é um operador diferencial de primeira ordem.

De fato, $D_{c}$ é $\mathbb{R}$-linear e

$$
\begin{aligned}
D_{c}(f V) & =\left(i_{f V} \mathrm{~d} \omega\right)(\dot{c})+\frac{\mathrm{d}}{\mathrm{d} t} i_{f V} \omega=\mathrm{d} \omega(f V, \dot{c})+\frac{\mathrm{d}}{\mathrm{d} t} \omega(f V) \\
& =f \mathrm{~d} \omega(V, \dot{c})+f \frac{\mathrm{d}}{\mathrm{d} t} \omega(V)+\frac{\mathrm{d} f}{\mathrm{~d} t} \omega(V)=f\left(\mathrm{~d} \omega(V, \dot{c})+\frac{\mathrm{d}}{\mathrm{d} t} \omega(V)\right)+\frac{\mathrm{d} f}{\mathrm{~d} t} \omega(V) \\
& =f D_{c}(V)+\frac{\mathrm{d} f}{\mathrm{~d} t} \omega(V) .
\end{aligned}
$$

Para escrever este operador em coordenadas tomemos uma vizinhança tubular de $c$ e um referencial ortonormal $\left\{e_{i}\right\}, 1 \leq i \leq 2 n$, de $c^{*}(D)$. Consideremos em $M$ a métrica tal que $\|\xi\|=1$ e $\xi \perp D$. Assim, $\left\{e_{i}, \xi\right\}$ é um referencial ortonormal ao longo de $c$. Sua base dual é $\left\{\theta^{i}, \omega\right\}$. 
Sendo $V \in C^{\infty}\left(c^{*}(T M)\right)$, temos $V=\sum_{i} v^{i} e_{i}+u \xi$, onde $v^{i}, u \in C^{\infty}([a, b], \mathbb{R})$.

Escrevemos $\dot{c} \in D$ como $\dot{c}=\sum_{i} a^{i} e_{i}$.

$$
\begin{aligned}
& \omega(V)=u \\
& \mathrm{~d} \omega=\sum_{i<k} h_{i j} \theta^{i} \wedge \theta^{j} \\
& \mathrm{~d} \omega(V, \dot{c})=\sum_{i<j} h_{i j} \theta^{i} \wedge \theta^{j}(V, \dot{c})=\sum_{i<j} h_{i j}\left(\theta^{i}(V) \theta^{j}(\dot{c})-\theta^{j}(V) \theta^{i}(\dot{c})\right) \\
& =\sum_{i<j} h_{i j}\left(v^{i} a^{j}-v^{j} a^{i}\right)=\sum_{i<j} h_{i j} a^{j} v^{i}-\sum_{i<j} h_{i j} a^{i} v^{j} \\
& =-\sum_{i<j} h_{j i} a^{j} v^{i}-\sum_{i<j} h_{i j} a^{i} v^{j}=-\sum_{i>j} h_{i j} a^{i} v^{j}-\sum_{i<j} h_{i j} a^{i} v^{j} \\
& =-\sum_{i, j} h_{i j} a^{i} v^{j} .
\end{aligned}
$$

Sendo $A^{j}=\sum_{i} h_{i j} a^{i}$, temos $\mathrm{d} \omega(V, \dot{c})=-\sum_{j} A^{j} v^{j}$ e, portanto,

$$
D_{c}(V)=\mathrm{d} \omega(V, \dot{c})+\frac{\mathrm{d}}{\mathrm{d} t} \omega(V)=-\sum_{j} A^{j} v^{j}+\frac{\mathrm{d} u}{\mathrm{~d} t},
$$

que é a expressão do operador $D_{c}$ em coordenadas.

Sendo $V$ uma variação infinitesimal de contato temos $V \in \operatorname{ker}\left(D_{c}\right)$, ou seja,

$$
\frac{\mathrm{d} u}{\mathrm{~d} t}=\sum_{j=1}^{2 n} A^{j} v^{j}
$$

e portanto

$$
u(t)=\sum_{j=1}^{2 n} \int_{a}^{t} A^{j}(x) v^{j}(x) \mathrm{d} x+u(a) .
$$

Para $V$ uma variação infinitesimal de contato com extremos fixos a condição $V(a)=$ $V(b)=0$ deve ser satisfeita. Como $V(a)=0$, temos a condição inicial $u(a)=0$ para a equação diferencial acima e assim o valor de $u(b)$ está determinado. Portanto, para $V$ ser uma variação infinitesimal de contato de extremos fixos, $u(b)=0$ é uma condição que $V$ deve satisfazer. Este fato motiva a definição de um operador.

Um campo $V$ ao longo de $c$ é um elemento de $C^{\infty}\left(c^{*}(D)\right) \oplus[\xi]$ e assim podemos escrevê-lo como $V=V^{H}+u \xi$, onde $V^{H}$ é um campo ao longo de $c \operatorname{com} V^{H}(t) \in D_{c(t)}$ e $u \in C^{\infty}([a, b], \mathbb{R})$. 
Sendo $V$ uma variação infinitesimal de contato escrevemos a condição $V \in \operatorname{ker}\left(D_{c}\right)$ como

$$
\frac{\mathrm{d} u}{\mathrm{~d} t}=A(t) V^{H}(t) .
$$

Para termos extremos fixos é necessário que $u(a)=0$ e $V^{H}(a)=V^{H}(b)=0$.

Seja $C_{0}^{\infty}\left(c^{*}(D)\right)$ o conjunto dos campos de vetores ao longo de $c$ tangentes a $D$ que se anulam em $a$ e $b$.

\section{Definição 2.18 Seja $H_{c}$ o operador}

$$
\begin{aligned}
H_{c}: C_{0}^{\infty}\left(c^{*}(D)\right) & \rightarrow \mathbb{R} \\
V^{H} & \rightarrow H_{c}\left(V^{H}\right)=\int_{a}^{b} A(t) V^{H}(t) \mathrm{d} t .
\end{aligned}
$$

Notemos que $H_{c}\left(V^{H}\right)=u(b)$, onde $u(b)$ é determinado pela equação diferencial $\frac{\mathrm{d} u}{\mathrm{~d} t}=$ $A(t) V^{H}(t)$ com condição inicial $u(a)=0$.

Proposição 2.4 operador $H_{c}$ é $\mathbb{R}$-linear.

De fato. Seja $u_{1}$ solução da equação diferencial $\frac{\mathrm{d} u_{1}}{\mathrm{~d} t}=A(t) V_{1}^{H}(t)$ com condição inicial $u_{1}(a)=0$.

Seja $u_{2}$ solução da equação diferencial $\frac{\mathrm{d} u_{2}}{\mathrm{~d} t}=A(t) V_{2}^{H}(t)$ com condição inicial $u_{2}(a)=0$ e $\lambda \in \mathbb{R}$.

$$
\begin{aligned}
\frac{\mathrm{d}}{\mathrm{d} t}\left(\lambda u_{1}+u_{2}\right) & =\lambda \frac{\mathrm{d} u_{1}}{\mathrm{~d} t}+\frac{\mathrm{d} u_{2}}{\mathrm{~d} t}=\lambda A(t) V_{1}^{H}(t)+A(t) V_{2}^{H}(t) \\
& =A(t)\left(\lambda V_{1}^{H}(t)+V_{2}^{H}(t)\right)
\end{aligned}
$$

e portanto $\lambda u_{1}+u_{2}$ é solução da equação diferencial $\frac{\mathrm{d} u}{\mathrm{~d} t}=A(t)\left(\lambda V_{1}^{H}(t)+V_{2}^{H}(t)\right)$ com condição inicial $u(0)=\left(\lambda u_{1}+u_{2}\right)(0)=\lambda u_{1}(0)+u_{2}(0)=0$. Assim,

$$
H_{c}\left(\lambda V_{1}^{H}+V_{2}^{H}\right)=u(b)=\lambda u_{1}(b)+u_{2}(b)=\lambda H_{c}\left(V_{1}^{H}\right)+H_{c}\left(V_{2}^{H}\right) .
$$

Definição 2.19 Dizemos que uma curva c é regular se $H_{c}: C_{0}^{\infty}\left(c^{*}(D)\right) \rightarrow \mathbb{R}$ é sobrejetor. 
Proposição 2.5 Seja c $:[a, b] \rightarrow M$ uma curva não constante, $c \in \Omega(D)$, onde $D$ é uma distribuição de contato. Então c é regular.

Basta mostrar que existe um campo $V^{H}$ para o qual $H_{c}\left(V^{H}\right) \neq 0$, isto é, que a equação diferencial $\frac{\mathrm{d} u}{\mathrm{~d} t}=A(t) V^{H}(t)$ com condição inicial $u(a)=0$ admite solução com $u(b) \neq 0$.

Vamos construir tal campo.

Existe $t_{0} \in(a, b)$ tal que $\dot{c}\left(t_{0}\right) \neq 0$, pois $c$ não é constante.

$\mathrm{d} \omega$ é não degenerada, assim

$$
\begin{aligned}
& i_{V} \mathrm{~d} \omega=0 \rightarrow V=0 \\
& \mathrm{~d} \omega=\sum_{i<j} h_{i j} \theta^{i} \wedge \theta^{j}=\sum_{i, j} h_{i j} \theta^{i} \otimes \theta^{j} \\
& i_{\dot{c}} \mathrm{~d} \omega=\sum_{i, j} h_{i j} \theta^{i}(\dot{c}) \theta^{j}=\sum_{j} A^{j} \theta^{j} \neq 0, \text { pois } \dot{c} \neq 0 \text { já que } \dot{c}\left(t_{0}\right) \neq 0 .
\end{aligned}
$$

Portanto, $\exists k A^{k} \neq 0$.

Seja $\bar{V}^{H}=A^{k} e_{k}$. Calculemos $H_{c}\left(\bar{V}^{H}\right)$.

$$
\begin{aligned}
& u(a)=0 \quad \text { e } \quad \frac{\mathrm{d} u}{\mathrm{~d} t}=\sum_{j} A^{j} v^{j}=\left(A^{k}\right)^{2} \\
& H_{c}\left(\bar{V}^{H}\right)=u(b)=u(a)+\int_{a}^{b}\left(A^{k}(t)\right)^{2} \mathrm{~d} t>0 .
\end{aligned}
$$

Decorre, imediatamente, a existência de $V^{H}$.

Proposição $2.6 c \in \Omega_{p q}(D), p \neq q \rightarrow c$ é regular.

Definição $2.20 \operatorname{ker}_{0}\left(D_{c}\right)$ é o conjunto das variações infinitesimais $V$ tais que $D_{c}(V)=0$ e $V(a)=V(b)=0$.

Lema 2.1 Dada uma vizinhança tubular de c e $\left\{e_{i}\right\}, 1 \leq i \leq 2 n+1$, um referencial ortonormal ao longo de $c$ de forma que $c\left(x_{1}\right)=\left(x_{1}, 0\right) \in \mathbb{R}^{2 n+1}$ e $\dot{c}\left(x_{1}\right)=e_{1}=\partial / \partial x_{1}$, existe um difeomorfismo $g$ tal que $g \circ c\left(x_{1}\right)=\left(x_{1}, 0\right)$ e $\mathrm{d} g\left(e_{i}\right)=\partial / \partial x_{i}, 1 \leq i \leq 2 n+1$.

Seja

$$
\begin{aligned}
& e_{i}\left(x_{1}\right)=\sum_{j=1}^{2 n+1} a_{i j}\left(x_{1}\right) \frac{\partial}{\partial x_{j}}, \quad 2 \leq i \leq 2 n+1 \\
& e_{1}\left(x_{1}\right)=\frac{\partial}{\partial x_{1}}
\end{aligned}
$$


Seja

$$
f\left(x_{1}, \ldots, x_{2 n+1}\right)=\sum_{i=2}^{2 n+1} \sum_{j=1}^{2 n+1} a_{i j}\left(x_{1}\right) x_{i} \frac{\partial}{\partial x_{j}}+x_{1} \frac{\partial}{\partial x_{1}} .
$$

Para $2 \leq i \leq 2 n+1$,

$$
\begin{aligned}
& \mathrm{d} f\left(\frac{\partial}{\partial x_{i}}\right)\left(x_{1}, 0\right)=\sum_{j=1}^{2 n+1} a_{i j}\left(x_{1}\right) \frac{\partial}{\partial x_{j}}=e_{i}, \\
& \mathrm{~d} f\left(\frac{\partial}{\partial x_{1}}\right)\left(x_{1}, 0\right)=\sum_{i=2}^{2 n+1} \sum_{j=1}^{2 n+1} \frac{\mathrm{d} a_{i j}}{\mathrm{~d} x_{1}} \cdot 0 \cdot \frac{\partial}{\partial x_{j}}+\frac{\partial}{\partial x_{1}}=\frac{\partial}{\partial x_{1}} .
\end{aligned}
$$

Basta tomar $g=f^{-1}$.

Proposição 2.7 Sejam $p, q \in M, p \neq q, c \in \Omega_{p q}(D)$. Então valem as implicações

$$
\begin{aligned}
& V \in \operatorname{ker}\left(D_{c}\right), V(a) \in D_{c(a)}, V(b) \in D_{c(b)} \rightarrow V \in T_{c} \Omega(D) \\
& V \in \operatorname{ker}_{0}\left(D_{c}\right) \rightarrow V \in T_{c} \Omega_{p q}(D) .
\end{aligned}
$$

Vamos construir uma variação de contato cuja variação infinitesimal é $V$.

Tomemos uma vizinhança tubular de $c$ difeomorfa ao espaço euclidiano onde $M=$ $\mathbb{R}^{2 n} \times \mathbb{R}$ de forma que a curva $c$ é dada por $c(t)=(t, 0), t \in[a, b]$ e $c^{*}(D)=[a, b] \times \mathbb{R}^{2 n}$ e $\left.\omega\right|_{c}=\mathrm{d} z$, onde $x_{1}, \ldots, x_{2 n}, z$ são as coordenadas de $\mathbb{R}^{2 n} \times \mathbb{R}$

$$
\begin{aligned}
& V=V^{H}+u \xi=V^{H}+u \frac{\partial}{\partial z} \\
& V^{H}(a)=V(a), V^{H}(b)=V(b), u(a)=u(b)=0 .
\end{aligned}
$$

Como $c$ é regular, podemos escolher $W^{H} \in C_{0}^{\infty}\left(c^{*}(D)\right)$ tal que $H_{c}\left(W^{H}\right) \neq 0$.

Seja $W \in \operatorname{ker}\left(D_{c}\right), W=W^{H}+w \partial / \partial z$. Seja $\Gamma$ dada por:

$$
\Gamma(t, s, \mu)=c(t)+s V^{H}(t)+\mu W^{H}(t) .
$$

Fixando $s$ e $\mu$ temos a curva em $\mathbb{R}^{2 n}$ dada por

$$
\begin{aligned}
& \gamma_{(s, \mu)}(t)=\Gamma(t, s, \mu) \\
& \gamma_{(s, \mu)}(a)=\Gamma(a, s, \mu)=c(a)+s V^{H}(a)+\mu W^{H}(a)=c(a)+s V^{H}(a) \\
& \gamma_{(s, \mu)}(b)=\Gamma(b, s, \mu)=c(b)+s V^{H}(b)+\mu W^{H}(b)=c(b)+s V^{H}(b)
\end{aligned}
$$


Seja $c_{(s, \mu)}:[a, b] \rightarrow \mathbb{R}^{2 n} \times \mathbb{R}$ o levantamento de $\gamma_{(s, \mu)}$ onde

$$
c_{(s, \mu)}(a)=c(a)+s V^{H}(a) \quad \text { e } \quad \omega\left(\dot{c}_{(s, \mu)}(t)\right)=0 .
$$

Coloquemos $\mathbf{C}(t, s, \mu)=c_{(s, \mu)}(t)$. $\mathbf{C}(t, s, \mu)$ é uma variação de $c$, pois $\mathbf{C}(t, 0,0)=c_{(0,0)}(t)$. $c_{(0,0)}(t)$ é o levantamento de $\gamma_{(0,0)}(t), \gamma_{(0,0)}(t)=\Gamma(t, 0,0)=c(t)$. Assim, $\omega\left(\dot{\gamma}_{(0,0)}(t)\right)=$ $\omega(\dot{c}(t))=0$ e $\gamma_{(0,0)}(a)=c(a)$ e portanto o levantamento de $\gamma_{(0,0)}(t)$ é a própria $\gamma_{(0,0)}(t)$. ou seja, $c_{(0,0)}(t)=\uparrow(0,0)(t)$. Assim,

$$
\mathbf{C}(t, 0,0)=c_{(0,0)}(t)=\gamma_{(0,0)}(t)=c(t)
$$

Podemos escrever $c_{(s, \mu)}$ na forma $\left(\gamma_{(s, \mu)}, z\right)$ e $\omega$ na forma $\omega=\mathrm{d} z-\sum_{i} a_{i} \mathrm{~d} x_{i}$, onde $a_{i}$. $1 \leq i \leq 2 n$, são funções $C^{\infty}$ de $x_{1}, \ldots, x_{2 n}, z$ e $a_{i}\left(x_{1}, 0\right)=0$.

A condição $c_{(s, \mu)}(a)=c(a)+s V^{H}(a)$ é então expressa por $z(a)=0$ e $\omega\left(\dot{c}_{(s, \mu)}\right)=0$ por

$$
\omega\left(\dot{\gamma}_{(s, \mu)}, \dot{z}\right)=0
$$

ou seja,

$$
\dot{z}=\sum a_{i} \mathrm{~d} x_{i}\left(\dot{\gamma}_{(s, \mu)}\right)
$$

Assim, existem $\Sigma_{1}$ e $\varepsilon_{2}$ positivos para os quais a equação diferencial acima com condição inicial $z(a)=0$ tem solução suave para $s, \mu \in\left(-\varepsilon_{1}, \varepsilon_{1}\right) \times\left(-\varepsilon_{2}, \varepsilon_{2}\right)$. Isto mostra a existência do levantamento $c_{(s . \mu)}$ (referência A.N. Tikonov, cap.II, §5).

Podemos escrever $\mathrm{C}(t, s, \mu)$ na forma

$$
\mathrm{C}(t, s, \mu)=(\Gamma(t, s, \mu), \alpha(t, s, \mu)) \text {. }
$$

Por construção, $\frac{\partial \mathbf{C}}{\partial s}(t, 0,0)$ é uma variação infinitesimal de $c$ que pertence a $\operatorname{ker}\left(D_{c}\right)$.

$$
\begin{aligned}
& \text { Como } \mathrm{C}(a, s, \mu)=c_{(s, \mu)}(a)=c(a)+s V^{H}(a)=c(a)+s V(a) \text {, temos } \\
& \frac{\partial \mathrm{C}}{\partial s}(a, 0,0)=V^{H}(a)=V(a) \in \mathbb{R}^{2 n} \rightarrow \frac{\partial \alpha}{\partial s}(a, 0,0)=0 \\
& \frac{\partial \mathbf{C}}{\partial s}(t, 0,0)=\frac{\partial \Gamma}{\partial s}(t, 0,0)+\frac{\partial \alpha}{\partial s}(t, 0,0) \frac{\partial}{\partial z}=V^{H}(t)+\frac{\partial \alpha}{\partial s}(t, 0,0) \frac{\partial}{\partial z} .
\end{aligned}
$$


Assim, $\frac{\partial \alpha}{\partial s}(t, 0,0)$ é solução da equação diferencial $\frac{\mathrm{d} u}{\mathrm{~d} t}=A(t) V^{H}(t)$ com condição inicial $u(a)=0$. Pela unicidade da solução temos

$$
\frac{\partial \alpha}{\partial s}(t, 0,0)=u(t)
$$

Analogamente, temos

$$
\frac{\partial \alpha}{\partial \mu}(t, 0,0)=w(t)
$$

Seja $\pi: \mathbb{R}^{2} \rightarrow \mathbb{R}$ definida por $\pi(s, \mu)=\alpha(b, s, \mu)$. Como $\alpha(t, 0,0)=\mathbf{C}(t, 0,0)-$ $\Gamma(t, 0,0)=c(t)-c(t)=0$, temos

$$
\begin{aligned}
\pi(0,0) & =\alpha(b, 0,0)=0 \\
\frac{\partial \pi}{\partial s}(0,0) & =\frac{\partial \alpha}{\partial s}(b, 0,0)=u(b)=0 \\
\frac{\partial \pi}{\partial \mu}(0,0) & =\frac{\partial \alpha}{\partial \mu}(b, 0,0)=w(b) \neq 0 .
\end{aligned}
$$

Pelo teorema da função implícita, a relação $\pi(s, \mu)=0$ determina $\mu$ como função de $s$ numa vizinhança de 0 .

Temos então $\mu=\mu(s) \operatorname{com} \mu(0)=0$ e $\pi(s, \mu(s))=0$,

$$
\begin{aligned}
& \frac{\mathrm{d}}{\mathrm{d} s} \pi(s, \mu(s))=\frac{\partial \pi}{\partial s}(s, \mu(s))+\frac{\partial \pi}{\partial \mu}(s, \mu(s)) \frac{\mathrm{d} \mu}{\mathrm{d} s}(s)=0, \\
& \frac{\partial \pi}{\partial s}(0,0)+\frac{\partial \pi}{\partial \mu}(0,0) \frac{\mathrm{d} \mu}{\mathrm{d} s}(0)=0 \rightarrow u(b)+w(b) \frac{\mathrm{d} \mu}{\mathrm{d} s}(0)=0 .
\end{aligned}
$$

$u(b)=0$ e $w(b) \neq 0, \log 0 \frac{\mathrm{d} \mu}{\mathrm{d} s}(0)=0$.

Seja

$$
\begin{aligned}
& \Gamma_{1}(t, s)=\mathbf{C}(t, s, \mu(s)) \\
& \Gamma_{1}(t, 0)=\mathbf{C}(t, 0, \mu(0))=\mathbf{C}(t, 0,0)=c(t) .
\end{aligned}
$$

Por construção, $\Gamma_{1}(t, s)$ é uma variação de contato de $c(t)$,

$$
\Gamma_{1}(a, s)=\mathrm{C}(a, s, \mu(s))=c_{(s, \mu(s))}(a)=c(a)+s V^{H}(a)=c(a)+s V(a),
$$




$$
\begin{aligned}
\Gamma_{1}(b, s) & =\mathrm{C}(b, s, \mu(s))=\Gamma(b, s, \mu(s))+\alpha(b, s, \mu(s)) \frac{\partial}{\partial z} \\
& =c(b)+s V^{H}(b)+\mu(s) W^{H}(b)+\alpha(b, s, \mu(s)) \frac{\partial}{\partial z} \\
& =c(b)+s V(b)+\alpha(b, s, \mu(s)) \frac{\partial}{\partial z}=c(b)+s V(b)+\pi(s, \mu(s)) \frac{\partial}{\partial z} \\
& =c(b)+s V(b) .
\end{aligned}
$$

Se $V(a)=V(b)=0$, então $\Gamma_{1}(a, s)=c(a)$ e $\Gamma_{1}(b, s)=c(b)$ e $\Gamma_{1}$ será uma variação de contato de $c$ com extremos fixos. Em geral,

$$
\begin{aligned}
\frac{\partial \Gamma_{1}}{\partial s}(a, 0) & =\left.\frac{\partial}{\partial s}(c(a)+s V(a))\right|_{s=0}=V(a) \\
\frac{\partial \Gamma_{1}}{\partial s}(b, 0) & =\left.\frac{\partial}{\partial s}(c(b)+s V(b))\right|_{s=0}=V(b), \\
\frac{\partial \Gamma_{1}}{\partial s}(t, 0) & =\frac{\partial \mathbf{C}}{\partial s}(t, 0,0)+\frac{\partial \mathbf{C}}{\partial \mu}(t, 0,0) \frac{\mathrm{d} \mu}{\mathrm{d} s}(0)=\frac{\partial \mathbf{C}}{\partial s}(t, 0,0) \\
& =V^{H}(t)+\frac{\partial \alpha}{\partial s}(t, 0,0) \frac{\partial}{\partial z}=V^{H}(t)+u(t) \frac{\partial}{\partial z}=V(t) .
\end{aligned}
$$

Assim, a variação infinitesimal associada a $\Gamma_{1}$, variação de contato de $c$ é $V(t)$, o que termina a demonstração.

Das proposições anteriores podemos caracterizar as variações infinitesimais de contato com extremos fixos no que segue.

Proposição 2.8 Seja $c \in \Omega_{p q}(D), p \neq q$. Então

$$
V \in T_{c} \Omega_{p q}(D) \leftrightarrow V \in \operatorname{ker}_{0}\left(D_{c}\right)
$$

Lema 2.2 A aplicação $I: F \rightarrow \mathbb{R}$

$$
f \rightarrow \int_{a}^{b} A(t) f(t) \mathrm{d} t
$$

onde $F=\left\{f \in C^{\infty}([a, b], \mathbb{R}) \mid f(a)=\right.$

$\left.K_{1}, f(b)=K_{2}\right\}, A \in C([a, b], \mathbb{R}), A \neq 0$, é sobrejetora.

Seja $\ell:[a, b] \rightarrow \mathbb{R}$ afim, $\ell(a)=K_{1}$ e $\ell(b)=K_{2}$. 
Seja $h=f-\ell, H=\left\{h \in C^{\infty}([a, b], \mathbb{R}) \mid h(a)=h(b)=0\right\}$ e $I_{1}$ o funcional linear definido por

$$
\begin{aligned}
I_{1}: H & \rightarrow \mathbb{R} \\
h & \rightarrow \int_{a}^{b} A(t) h(t) \mathrm{d} t .
\end{aligned}
$$

Como

$$
I(f)=\int_{a}^{b} A f \mathrm{~d} t=\int_{a}^{b} A(\ell+h) \mathrm{d} t=\int_{a}^{b} A \ell \mathrm{d} t+I_{1}(h),
$$

basta mostrar que $I_{1}$ é sobrejetor.

$A \neq 0$. Assim, $\exists J=\left[a_{1}, b_{1}\right] \subset[a, b] \forall x \forall y x, y \in J A(x) A(y)>0$. Podemos assumir $\forall x \in J, A(x)>0$.

Seja $\tau \in C^{\infty}([a, b], \mathbb{R})$ tal que

$$
\begin{aligned}
& \forall x \in[a, b], \tau(x) \geq 0, \\
& \forall x \in[a, b]-J, \tau(x)=0, \\
& \tau\left(a_{1}\right)=\tau\left(b_{1}\right)=0, \\
& \forall x \in\left[\frac{3 a_{1}+b_{1}}{4}, \frac{a_{1}+3 b_{1}}{4}\right], \quad \tau(x)=1 .
\end{aligned}
$$

Seja $m=\min \{A(x) \mid x \in J\}$

$$
\begin{aligned}
I_{1}(\tau) & =\int_{a}^{b} A \tau \mathrm{d} t=\int_{a_{1}}^{b_{1}} A \tau \mathrm{d} t=\int_{a_{1}}^{\frac{3 a_{1}+b_{1}}{4}} A \tau \mathrm{d} t+\int_{\frac{3 a_{1}+b_{1}}{4}}^{\frac{a_{1}+3 b_{1}}{4}} A \tau \mathrm{d} t+\int_{\frac{a_{1}+3 b_{1}}{4}}^{b_{1}} A \tau \mathrm{d} t \\
& \geq \int_{\frac{3 a_{1}+b_{1}}{4}}^{\frac{a_{1}+3 b_{1}}{4}} A \tau \mathrm{d} t \geq m \frac{\left(b_{1}-a_{1}\right)}{2}>0 .
\end{aligned}
$$

Como $I_{1}(\tau)>0, I_{1}$ é sobrejetor, o que demonstra o lema.

Proposição 2.9 Seja $c \in \Omega_{p q}(D), p \neq q$, e $V_{1} \in D_{p}, V_{2} \in D_{q}$. Então existe uma variação infinitesimal de contato $V \in T_{c} \Omega(D)$ tal que $V(a)=V_{1}$ e $V(b)=V_{2}$.

Pela Proposição 2.7 basta mostrar que existe uma variação infinitesimal $V$ tal que $V(a)=V_{1}, V(b)=V_{2}$ e $V \in \operatorname{ker}\left(D_{c}\right)$. 
Utilizando a expressão do operador $D_{c}$ em coordenadas a condição $V \in \operatorname{ker} D_{c}$ é dada por

$$
u(t)=\sum_{j=1}^{2 n} \int_{a}^{t} A^{j}(x) v^{j}(x) \mathrm{d} x+u(a)
$$

e temos as condições $u(a)=u(b)=0$ para a variação infinitesimal $V$.

Assim, as condições sobre $V$ são dadas por

$$
\sum_{j=1}^{2 n} \int_{a}^{b} A^{j}(t) v^{j}(t) \mathrm{d} t=0
$$

Como $c$ é regular existe $k, A^{k} \neq 0$.

Escrevamos a igualdade anterior como

$$
\int_{a}^{b} A^{k}(t) v^{k}(t) \mathrm{d} t=\sum_{j \neq k} \int_{a}^{b} A^{j}(t) v^{j}(t) \mathrm{d} t
$$

Escolhamos arbitrariamente funções $w^{j}$ satisfazendo $w^{j}(a)=v_{1}^{j}, w^{j}(b)=v_{2}^{j}$, para $1 \leq$ $j \leq 2 n, j \neq k$. Seja

$$
m=\sum_{j \neq k} \int_{a}^{b} A^{j}(t) w^{j}(t) \mathrm{d} t \quad \text { e } \quad F=\left\{f \in C^{\infty}([a, b], \mathbb{R}) \mid f(a)=v_{1}^{k}, f(b)=v_{2}^{k}\right\} .
$$

Seja $I$ a aplicação $I: F \rightarrow \mathbb{R}$

$$
f \rightarrow \int_{a}^{b} A^{k}(t) f(t) \mathrm{d} t
$$

Como $I$ é sobrejetora existe $w^{k} \in F, I\left(w^{k}\right)=m$. Assim, a variação infinitesimal

$$
V(t)=\sum_{j=1}^{2 n} w^{j}(t) e_{j}(t)+u(t) \xi(t)
$$

onde $u(t)=\sum_{j=1}^{2 n} \int_{a}^{t} A^{j}(x) w^{j}(x) \mathrm{d} x$ satisfaz $V(a)=V_{1}, V(b)=V_{2}$ e $V \in \operatorname{ker}\left(D_{c}\right)$, o que termina a demonstração.

Nosso objetivo agora é estudar o funcional energia de modo a determinar as suas curvas extremais as quais serão chamadas de geodésicas. 
Teorema 2.2 (Primeira Variação da Energia) Seja $c \in \Omega_{p q}(D), p \neq q$, suave por partes na partição $P=\left\{a=t_{0}, \ldots, t_{l}=b\right\}$ e $\Gamma$ uma variação de contato de c cuja variação infinitesimal é $V$. Então, $\mathrm{d} E_{c}: T_{c} \Omega(D) \rightarrow \mathbb{R}$ é dada por

$$
\begin{aligned}
\mathrm{d} E_{c}(V)= & \langle V(b), \dot{c}(b)\rangle-\langle V(a), \dot{c}(a)\rangle-\sum_{i=1}^{l-1}\left\langle V\left(t_{i}\right), \dot{c}\left(t_{i}^{+}\right)-\dot{c}\left(t_{i}^{-}\right)\right\rangle \\
& -\int_{a}^{b}\left\langle\nabla_{\dot{c}} \dot{c}-\langle\tau(\dot{c}), \dot{c}\rangle \xi, V\right\rangle \mathrm{d} t,
\end{aligned}
$$

onde $\dot{c}(a)=\dot{c}\left(t_{0}^{+}\right)$e $\dot{c}(b)=c\left(t_{l}^{-}\right)$.

De fato,

$$
\begin{aligned}
& E(c)=\frac{1}{2} \int_{a}^{b} g(\dot{c}, \dot{c}) \mathrm{d} t=\frac{1}{2} \int_{a}^{b}\langle\dot{c}, \dot{c}\rangle \mathrm{d} t \\
& \mathrm{~d} E_{c}(V)=\left.\frac{\mathrm{d}}{\mathrm{d} s} E\left(c_{s}\right)\right|_{s=0}=\left.\frac{\mathrm{d}}{\mathrm{d} s}\left(\frac{1}{2} \int_{a}^{b}\left\langle\dot{c}_{s}, \dot{c}_{s}\right\rangle \mathrm{d} t\right)\right|_{s=0} \\
& =\left.\frac{\mathrm{d}}{\mathrm{d} s}\left(\frac{1}{2} \int_{a}^{b}\langle T, T\rangle \mathrm{d} t\right)\right|_{s=0}=\left.\frac{1}{2} \int_{a}^{b} V\langle T, T\rangle \mathrm{d} t\right|_{s=0} \\
& =\left.\frac{1}{2} \sum_{i=0}^{l-1} \int_{t_{i}}^{t_{i+1}} V\langle T, T\rangle \mathrm{d} t\right|_{s=0} \\
& V\langle T, T\rangle=2\left\langle\nabla_{V} T, T\right\rangle \\
& \nabla_{V} T-\nabla_{T} V-[V, T]=T_{o r}(V, T), \quad[V, T]=0 \\
& \nabla_{V} T=T_{o r}(V, T)+\nabla_{T} V \\
& V\langle T, T\rangle=2\left\langle\nabla_{T} V+T_{\text {or }}(V, T), T\right\rangle=2\left(\left\langle\nabla_{T} V, T\right\rangle+\left\langle T_{\text {or }}(V, T), T\right\rangle\right) \\
& \frac{\mathrm{d}}{\mathrm{d} t}\langle V, T\rangle=T\langle V, T\rangle=\left\langle\nabla_{T} V, T\right\rangle+\left\langle V, \nabla_{T} T\right\rangle \\
& V\langle T, T\rangle=2\left(\frac{\mathrm{d}}{\mathrm{d} t}\langle V, T\rangle-\left\langle V, \nabla_{T} T\right\rangle+\left\langle T_{o r}(V, T), T\right\rangle\right) \\
& \mathrm{d} E_{c}(V)=\sum_{i=0}^{l-1} \int_{t_{i}}^{t_{i+1}} \frac{\mathrm{d}}{\mathrm{d} t}\langle V, T\rangle-\left\langle V, \nabla_{T} T\right\rangle+\left.\left\langle T_{o r}(V, T), T\right\rangle \mathrm{d} t\right|_{s=0} \\
& =\left.\left.\sum_{i=1}^{l-1}\langle V, T\rangle\right|_{t_{i}} ^{t_{i+1}}\right|_{s=0}-\sum_{i=0}^{l-1} \int_{t_{i}}^{t_{i+1}}\left\langle V, \nabla_{T} T\right\rangle-\left.\left\langle T_{o r}(V, T), T\right\rangle \mathrm{d} t\right|_{s=0} \\
& =\left.\sum_{i=0}^{l-1}\langle V, \dot{c}\rangle\right|_{t_{i}} ^{t_{i+1}}-\int_{a}^{b}\left\langle V, \nabla_{\dot{c}} \dot{c}\right\rangle-\left\langle T_{o r}(V, \dot{c}), \dot{c}\right\rangle \mathrm{d} t
\end{aligned}
$$




$$
\begin{aligned}
& \left.\sum_{i=0}^{l-1}\langle V, \dot{c}\rangle\right|_{t_{i}} ^{t_{i+1}}=\left\langle V\left(t_{l}\right), \dot{c}\left(t_{l}^{-}\right)\right\rangle-\left\langle V\left(t_{0}\right), \dot{c}\left(t_{0}^{+}\right)\right\rangle-\sum_{i=1}^{l-1}\left\langle V\left(t_{i}\right), \dot{c}\left(t_{i}^{+}\right)-\dot{c}\left(t_{i}^{-}\right)\right\rangle \\
& =\langle V(b), \dot{c}(b)\rangle-\langle V(a), \dot{c}(a)\rangle-\sum_{i=1}^{l-1}\left\langle V\left(t_{i}\right), \dot{c}\left(t_{i}^{+}\right)-\dot{c}\left(t_{i}^{-}\right)\right\rangle \\
& T_{o r}(V, \dot{c})=(\omega \wedge \tau+\mathrm{d} \omega \otimes \xi)(V, \dot{c})=\omega(V) \tau(\dot{c})-\omega(\dot{c}) \tau(V)+\mathrm{d} \omega(V, \dot{c}) \xi \\
& \left\langle T_{o r}(V, \dot{c}), \dot{c}\right\rangle=\omega(V)\langle\tau(\dot{c}), \dot{c}\rangle-\omega(\dot{c})\langle\tau(V), \dot{c}\rangle+\mathrm{d} \omega(V, \dot{c})\langle\xi, \dot{c}\rangle \\
& =\omega(V)\langle\tau(\dot{c}), \dot{c}\rangle-\omega(\dot{c})\langle\tau(V), \dot{c}\rangle+\omega(\dot{c}) \mathrm{d} \omega(V, \dot{c}) \\
& =\langle\tau(\dot{c}), \dot{c}\rangle\langle\xi, V\rangle-\omega(\dot{c})(\langle\tau(V), \dot{c}\rangle-\mathrm{d} \omega(V, \dot{c})) .
\end{aligned}
$$

Como $c \in \Omega(D), \omega(\dot{c})=0$, portanto

$$
\left\langle T_{\text {or }}(V, \dot{c}), \dot{c}\right\rangle=\langle\langle\tau(c), \dot{c}\rangle \xi, V\rangle
$$

Assim,

$$
\begin{aligned}
\mathrm{d} E_{c}(V)= & \langle V(b), \dot{c}(b)\rangle-\langle V(a), \dot{c}(a)\rangle-\sum_{i=1}^{l-1}\left\langle V\left(t_{i}\right), \dot{c}\left(t_{i}^{+}\right)-\dot{c}\left(t_{i}^{-}\right)\right\rangle \\
& -\int_{a}^{b}\left\langle\nabla_{\dot{c}} \dot{c}-\langle\tau(\dot{c}), \dot{c}\rangle \xi, V\right\rangle \mathrm{d} t .
\end{aligned}
$$

Proposição 2.10 Seja $c \in \Omega_{p q}(D), p \neq q$, suave por partes na partição $\left\{a=t_{0}, \ldots, t_{l}=\right.$ b\} e V uma variação infinitesimal de contato de extremos fixos. Então

$$
\mathrm{d} E_{c}: T_{c} \Omega_{p q}(D) \rightarrow \mathbb{R}
$$

é dada por

$$
\mathrm{d} E_{c}(V)=-\sum_{i=1}^{l-1}\left\langle V\left(t_{i}\right), \dot{c}\left(t_{i}^{+}\right)-\dot{c}\left(t_{i}^{-}\right)\right\rangle-\int_{a}^{b}\left\langle\nabla_{\dot{c}} \dot{c}-\langle\tau(\dot{c}), \dot{c}\rangle \xi, V\right\rangle \mathrm{d} t .
$$

De fato, basta fazer $V(a)=V(b)=0$ no Teorema 2.2 .

A seguir vamos demonstrar um teorema que caracteriza as curvas suaves que são extremos do funcional energia. Para tanto faremos uso do seguinte lema. 
Lema 2.3 Sejam $A:[a, b] \rightarrow \mathbb{R}^{n}$ e $\Lambda:[a, b] \rightarrow \mathbb{R}^{n}$ suaves tal que a aplicação

$$
\begin{aligned}
& H: C_{0}^{\infty}\left([a, b], \mathbb{R}^{n}\right) \rightarrow \mathbb{R} \\
& H(w)=\int_{a}^{b} A(t) w(t) \mathrm{d} t
\end{aligned}
$$

é sobrejetora. Então

$$
\left(H(w)=0 \rightarrow \int_{a}^{b} \Lambda(t) w(t) \mathrm{d} t=0\right) \rightarrow \exists k \in \mathbb{R} \Lambda=k A
$$

De fato, como $H$ é sobrejetora, temos $A \neq 0$. Portanto $\int_{a}^{b}\|A(t)\|^{2} \mathrm{~d} t>0$.

Seja $k \in \mathbb{R}$ solução da equação

$$
\int_{a}^{b} A(t) \Lambda(t) \mathrm{d} t-k \int_{a}^{b}\|A(t)\|^{2} \mathrm{~d} t=0
$$

Assim,

$$
\int_{a}^{b} A(t)(\Lambda(t)-k A(t)) \mathrm{d} t=0
$$

Seja $w(t)=\Lambda(t)-k A(t)$.

A igualdade acima é então escrita como $H(w)=0$.

Calculemos agora $\int_{a}^{b}\|\Lambda(t)-k \cdot A(t)\|^{2} \mathrm{~d} t$.

$$
\begin{aligned}
& \int_{a}^{b}\|\Lambda(t)-k \cdot A(t)\|^{2} \mathrm{~d} t=\int_{a}^{b}(\Lambda(t)-k: A(t)) w(t) \mathrm{d} t \\
& =\int_{a}^{b} \Lambda(t) w(t) \mathrm{d} t-k \cdot \int_{a}^{b} A(t) w(t) \mathrm{d} t=\int_{a}^{b} \Lambda(t) w(t) \mathrm{d} t-k \cdot H(w) .
\end{aligned}
$$

$H(w)=0$ por construção e por hipótese $H(w)=0 \rightarrow \int_{a}^{b} \Lambda(t) w(t) \mathrm{d} t=0$. Assim,

$$
\int_{a}^{b}\|\Lambda(t)-k \cdot A(t)\|^{2} \mathrm{~d} t=0
$$

Logo, $\Lambda=k \cdot A$.

Para o teorema que segue, lembramos que $h$ é definido por $\mathrm{d} w(X, Y)=\langle h(X), Y\rangle$.

Teorema 2.3 Uma curva suave $c \in \Omega_{p q}(D), p \neq q$, é extremo de $E$ se e somente se

$$
\nabla_{\dot{c}} \dot{c}=-\left(\int_{a}^{t}\langle\tau(\dot{c}), \dot{c}\rangle \mathrm{d} x-k\right) h(\dot{c})
$$


Demonstração: Se $\nabla_{\dot{c}} \dot{c}=-\left(\int_{a}^{t}\langle\tau(\dot{c}), \dot{c}\rangle \mathrm{d} x-k\right) h(\dot{c})$ e $\omega(\dot{c})=0$, então

$$
\begin{aligned}
\mathrm{d} E_{c}(V) & =\int_{a}^{b}\left\langle\langle\tau(\dot{c}), \dot{c}\rangle \xi-\nabla_{\dot{c}} \dot{c}, V\right\rangle \mathrm{d} t \\
& =\int_{a}^{b}\left\langle\langle\tau(\dot{c}), \dot{c}\rangle \xi+\left(\int_{a}^{t}\langle\tau(\dot{c}), \dot{c}\rangle \mathrm{d} x-k\right) h(\dot{c}), V\right\rangle \mathrm{d} t
\end{aligned}
$$

$h(\dot{c})=\sum_{k} A^{k} e_{k}=A, V=\sum_{k} v^{k} e_{k}+u \xi$. Assim,

$$
\langle h(\dot{c}), V\rangle=\sum_{k} A^{k} v^{k}
$$

O integrando da expressão de $\mathrm{d} E_{c}(V)$ fica

$$
u\langle\tau(\dot{c}), \dot{c}\rangle+\left(\int_{a}^{t}\langle\tau(\dot{c}), \dot{c}\rangle \mathrm{d} x-k\right) \sum_{k} A^{k} v^{k}
$$

Se $V \in \operatorname{ker}_{0}\left(D_{c}\right)$, então $u(t)=\int_{a}^{t} \sum_{k} A^{k} v^{k} \mathrm{~d} x$ e $u(b)=u(a)=0$.

Chamemos

$$
\begin{aligned}
f(t) & =\int_{a}^{t}\langle\tau(\dot{c}), \dot{c}\rangle \mathrm{d} x-k, \\
f^{\prime}(t) & =\langle\tau(\dot{c}), \dot{c}\rangle .
\end{aligned}
$$

Assim, o integrando será escrito $u(t) f^{\prime}(t)+f(t) u^{\prime}(t)$ e

$$
\begin{aligned}
\mathrm{d} E_{c}(V) & =\int_{a}^{b} u(t) f^{\prime}(t)+f(t) u^{\prime}(t) \mathrm{d} t=\int_{a}^{b} \frac{\mathrm{d}}{\mathrm{d} t}(u . f) \mathrm{d} t \\
& =\left.u \cdot\right|_{a} ^{b}=u(b) f(b)-u(a) f(a)=0 . f(b)-0 . f(a)=0
\end{aligned}
$$

e portanto $c$ é extremo de $E$.

Se $c$ é extremo de $E$, temos

$$
\begin{aligned}
& \forall V \in T_{c} \Omega_{p q}(D)=\operatorname{ker}_{0}\left(D_{c}\right), \quad \mathrm{d} E_{c}(V)=\int_{a}^{b}\left\langle-\nabla_{\dot{c}} \dot{c}+\langle\tau(\dot{c}), \dot{c}\rangle \xi, V\right\rangle \mathrm{d} t=0 \\
& \mathrm{~d} E_{c}(V)=\int_{a}^{b}\left\langle-\nabla_{\dot{c}} \dot{c}, V^{H}\right\rangle+u\langle\tau(\dot{c}), \dot{c}\rangle \mathrm{d} t
\end{aligned}
$$

Coloquemos $f^{\prime}(t)=\langle\tau(\dot{c}), \dot{c}\rangle, f(t)=\int_{a}^{t}\langle\tau(\dot{c}), \dot{c}\rangle \mathrm{d} x+k_{1}$,

$$
\int_{a}^{b} u\langle\tau(\dot{c}), \dot{c}\rangle \mathrm{d} t=\left.u f\right|_{a} ^{b}-\int_{a}^{b} f \frac{\mathrm{d} u}{\mathrm{~d} t} \mathrm{~d} t=\left.u f\right|_{a} ^{b}-\int_{a}^{b} f A V^{H} \mathrm{~d} t .
$$


Logo,

$$
\begin{aligned}
& \mathrm{d} E_{c}(V)=\int_{a}^{b}-\left\langle\nabla_{\dot{c}} \dot{c}+f A, V^{H}\right\rangle \mathrm{d} t+\left.u f\right|_{a} ^{b}, \\
& u(a)=0 \quad \text { e } \quad u(b)=\int_{a}^{b} A V^{H} \mathrm{~d} t=H_{c}\left(V^{H}\right) .
\end{aligned}
$$

Assim,

$$
\forall V \in T_{c} \Omega_{p q}(D), \quad \mathrm{d} E_{c}(V)=\int_{a}^{b}-\left\langle\nabla_{\dot{c}} \dot{c}+f A, V^{H}\right\rangle \mathrm{d} t+f(b) H_{c}\left(V^{H}\right)=0 .
$$

Como $c$ é regular, $H_{c}$ é sobrejetor.

Pela igualdade acima, se $H_{c}\left(V^{H}\right)=0$, então $\int_{a}^{b}-\left\langle\nabla_{\dot{c}} \dot{c}+f A, V^{H}\right\rangle \mathrm{d} t=0$.

Se $L \in C_{0}^{\infty}\left([a, b], \mathbb{R}^{2 n}\right), H_{c}(L)=0$, então $W=L+l \xi$, onde $l=\int_{a}^{t} A L \mathrm{~d} t$ pertence a $T_{c} \Omega_{p q}(D)=\operatorname{ker}_{0}\left(D_{c}\right)$.

Como

$$
\forall V \in T_{c} \Omega_{p q}(D) \quad H_{c}\left(V^{H}\right)=u(b)=0,
$$

estamos nas condições do lema anterior e portanto existe $k_{2} \in \mathbb{R}$ tal que

$$
\begin{aligned}
& -\left(\nabla_{\dot{c}} \dot{c}+f A\right)=k_{2} A \\
& \nabla_{\dot{c}} \dot{c}=-\left(f+k_{2}\right) A, \quad \text { ou seja, } \\
& \nabla_{\dot{c}} \dot{c}=-\left(f(t)+k_{2}\right) h(\dot{c}) \quad \text { e } \\
& \nabla_{\dot{c} \dot{c}}=-\left(\int_{a}^{t}\langle\tau(\dot{c}), \dot{c}\rangle \mathrm{d} x-k\right) h(\dot{c}), \quad \text { onde } k=-\left(k_{1}+k_{2}\right) .
\end{aligned}
$$

Corolário 2.1 Seja $c \in \Omega_{p q}(D)$ uma curva suave tal que

$$
\nabla_{\dot{c}} \dot{c}=-\left(\int_{a}^{t}\langle\tau(\dot{c}), \dot{c}\rangle \mathrm{d} x-k\right) h(\dot{c}) .
$$

Então $\int_{a}^{b}\left\langle\langle\tau(\dot{c}), \dot{c}\rangle \xi-\nabla_{\dot{c}} \dot{c}, V\right\rangle \mathrm{d} t=0$ para todo $V \in \operatorname{ker}\left(D_{c}\right)$ tal que $V(a) \in D_{c(a)}$ e $V(b) \in$ $D_{c}(b)$. 
De fato, basta notar que na primeira parte da demonstração do Teorema 2.3 só utilizamos as condições $u(a)=u(b)=0$ e $\frac{\mathrm{d} u}{\mathrm{~d} t}=\sum_{k} A^{k} v^{k}$ para concluirmos que $\int_{a}^{b}\langle\langle\tau(\dot{c}) . \dot{c}\rangle \xi-$ $\left.\nabla_{\dot{c}} \dot{c} . I^{\top}\right\rangle \mathrm{d} t=0$ e estas condições são satisfeitas por $V$.

Corolário 2.2 Seja $c \in \Omega_{p q}(D)$ nas condições do Corolário 2.1 e $V \in T_{c} \Omega(D)$ tal que $V(a) \in D_{c(a)}$ e $V(b) \in D_{c}(b)$. Então

$$
\mathrm{d} E_{c}(V)=\langle V(b), \dot{c}(b)\rangle-\langle V(a), \dot{c}(a)\rangle .
$$

Demonstração: Imediata do Teorema 2.2 e Corolário 2.1.

Teorema 2.4 Seja $c \in \Omega_{p q}(D), p \neq q$, suave por partes na partição $P=\left\{a=t_{0}, \ldots, t_{l}=\right.$ b) um extremo de E. Então c é suave.

Demonstração: Seja $c_{i}=\left.c\right|_{\left[t_{i}, t_{i+1}\right]}, 0 \leq i \leq l-1$.

Seja $V_{i} \in T_{c_{i}} \Omega_{c\left(t_{i}\right) c\left(t_{i+1}\right)}(D)$ e $\bar{V}_{i} \in T_{c} \Omega_{p q}(D)$ tal que $\bar{V}_{i}(t)=0$ para $t \in[a, b]-\left(t_{i}, t_{i+1}\right)$ e $\bar{V}_{i}(t)=V(t)$ para $t \in\left(t_{i}, t_{i+1}\right)$.

Do Teorema 2.2 temos $\mathrm{d} E_{c_{i}}\left(V_{i}\right)=\mathrm{d} E_{c}\left(\bar{V}_{i}\right)$.

Como $c$ é extremo de $E$, temos $\mathrm{d} E_{c}\left(\bar{V}_{i}\right)=0$. Logo, $\mathrm{d} E_{c_{i}}\left(V_{i}\right)=0$ e portanto $c_{i}$ é uma curva suave extremo de $E$.

Pelo Teorema 2.3, $c_{i}$ satisfaz a equação

$$
\nabla_{\dot{c}_{i}} \dot{c}_{i}=-\left(\int_{t_{i}}^{t}\left\langle\tau\left(\dot{c}_{i}\right), \dot{c}_{i}\right\rangle \mathrm{d} x-k_{i}\right) h\left(\dot{c}_{i}\right)
$$

Tomemos agora $W$ uma variação infinitesimal de contato de $c$ tal que $W(a)=W(b)=$ 0 e $W\left(t_{i}\right)=\dot{c}\left(t_{i}^{+}\right)-\dot{c}\left(t_{i}^{-}\right)$para $1 \leq i \leq l-1$. A existência de tal variação é garantida pela Proposição 2.9.

Como $c$ é extremo de $E$, temos

$$
\left.\mathrm{d} E_{c}(W)=-\sum_{i=1}^{l-1}\left|\dot{c}\left(t_{i}^{+}\right)-\dot{c}\left(t_{i}^{-}\right)\right|^{2}-\int_{a}^{b} \nabla_{\dot{c}} \dot{c}-\langle\tau(\dot{c}), \dot{c}\rangle \xi, W\right\rangle \mathrm{d} t=0 .
$$


Seja $W_{i}=\left.W\right|_{\left[t_{i}, t_{i+1}\right]}$.

As condições do Corolário 2.1 são satisfeitas por $c_{i}$ e $W_{i}$ e portanto

$$
\int_{t_{i}}^{t_{i+1}}\left\langle\nabla_{\dot{c}_{i}} \dot{c}_{i}-\left\langle\tau\left(\dot{c}_{i}\right), \dot{c}_{i}\right\rangle \xi, W_{i}\right\rangle \mathrm{d} t=0
$$

Assim, $\int_{a}^{b}\left\langle\nabla_{\dot{c}} \dot{c}-\langle\tau(\dot{c}), \dot{c}\rangle \xi, W\right\rangle \mathrm{d} t=0$.

Logo, $\mathrm{d} E_{c}(W)=-\sum_{i=1}^{l-1}\left|\dot{c}\left(t_{i}^{+}\right)-\dot{c}\left(t_{i}^{-}\right)\right|^{2}=0$.

Da igualdade acima, temos $\dot{c}\left(t_{i}^{+}\right)=\dot{c}\left(t_{i}^{-}\right)$e portanto $c$ é diferenciável em $t_{i}, 1 \leq i \leq$ $l-1$.

$$
\nabla_{\dot{c}\left(t_{i}^{-}\right)} \dot{c}\left(t_{i}^{-}\right)=\nabla_{\dot{c}\left(t_{i}^{+}\right)} \dot{c}\left(t_{i}^{+}\right) \rightarrow \nabla_{\dot{c}_{i-1}\left(t_{i}\right)} \dot{c}_{i-1}\left(t_{i}\right)=\nabla_{\dot{c}_{i}\left(t_{i}\right)} \dot{c}_{i}\left(t_{i}\right)
$$

Da expressão de $\nabla_{\dot{c}_{i}} \dot{c}_{i}$, temos

$$
\int_{t_{i-1}}^{t_{i}}\langle\tau(\dot{c}), \dot{c}\rangle \mathrm{d} x-k_{i-1}=\int_{t_{i}}^{t_{i}}\langle\tau(\dot{c}), \dot{c}\rangle \mathrm{d} x-k_{i}=-k_{i}
$$

Assim, para $t \in\left[t_{i}, t_{i+1}\right]$,

$$
\nabla_{\dot{c}} \dot{c}=-\left(\int_{t_{i}}^{t}\langle\tau(\dot{c}), \dot{c}\rangle \mathrm{d} x-k_{i}\right) h(\dot{c})=-\left(\int_{t_{i-1}}^{t}\langle\tau(\dot{c}), \dot{c}\rangle \mathrm{d} x-k_{i-1}\right) h(\dot{c})
$$

e, repetindo o argumento, diminuímos o índice $i$ de forma a obtermos

$$
\nabla_{\dot{c}} \dot{c}=-\left(\int_{t_{0}=a}^{t}\langle\tau(\dot{c}), \dot{c}\rangle \mathrm{d} x-k_{0}\right) h(\dot{c})
$$

Como $c$ satisfaz à equação acima, ela é suave. 


\section{Capítulo 3}

\section{Exemplos}

Neste capítulo vamos apresentar três exemplos de variedades sub-riemannianas de contato.

Estudaremos as geodésicas e a aplicação exponencial nestas variedades.

\section{Exemplo 1: Espaço de Heisenberg}

Seja $D$ a distribuição gerada pelos campos $e_{1}$ e $e_{2}$ no $\mathbb{R}^{3}$,

$$
\begin{aligned}
& e_{1}=\frac{\partial}{\partial x}-y \frac{\partial}{\partial z} \\
& e_{2}=\frac{\partial}{\partial y}+x \frac{\partial}{\partial z}
\end{aligned}
$$

Como $\left[e_{1}, e_{2}\right]=2 \frac{\partial}{\partial z}$, a matriz da aplicação

$$
\begin{aligned}
L: D \times D & \rightarrow T \mathbb{R}^{3} / D \\
(X, Y) & \rightarrow[X, Y] \bmod D
\end{aligned}
$$

na base $\left\{e_{1}, e_{2}, \partial / \partial z\right\}$ é $\left[\begin{array}{cc}0 & 2 \\ -2 & 0\end{array}\right]$ e portanto $L$ é não degenerada.

Assim, já que $\operatorname{dim} D=2$, a distribuição $D$ é de contato.

Em $D$ vamos definir uma métrica de forma que a projeção

$$
\begin{aligned}
\pi: \mathbb{R}^{3} & \rightarrow \mathbb{R}^{2} \\
(x, y, z) & \rightarrow(x, y)
\end{aligned}
$$


seja uma isometria sobre $D$.

Como $\mathrm{d} \pi\left(e_{1}\right)=\frac{\partial}{\partial x}$ e $\mathrm{d} \pi\left(e_{2}\right)=\frac{\partial}{\partial y}$, a métrica em $D$ fica definida impondo que $\left\{e_{1}, \epsilon_{2}\right\}$ seja uma base ortonormal.

Observamos que $\mathrm{d} x\left(e_{1}\right)=1, \mathrm{~d} x\left(e_{2}\right)=0, \mathrm{~d} y\left(e_{1}\right)=0 \mathrm{e} \mathrm{d} y\left(e_{2}\right)=1$ e portanto a restrição das diferenciais d $x$ e dy a $D$ formam uma base dual de $\left\{e_{1}, e_{2}\right\}$. Assim, podemos escrever a métrica em $D$ na forma $g=\mathrm{d} x^{2}+\mathrm{d} y^{2}$.

A variedade sub-riemanniana de contato $\left(\mathbb{R}^{3}, D, g\right)$ é chamada de espaço de Heisemberg de dimensão 3 .

O elemento de área em $D$ é a forma $\mathrm{d} A=\mathrm{d} x \wedge \mathrm{d} y$ sobre $D$, pois $\mathrm{d} A\left(e_{1}, e_{2}\right)=1$.

Vamos determinar a forma de contato $\omega$.

Como $\operatorname{dim} M=3$, temos as condições

$$
\operatorname{ker} \omega=D \quad \text { e }\left.\quad \mathrm{d} \omega\right|_{D}=2 \mathrm{~d} A .
$$

Assim, $\omega\left(e_{1}\right)=\omega\left(e_{2}\right)=0$ e $\left.\mathrm{d} \omega\right|_{D}=2 \mathrm{~d} x \wedge \mathrm{d} y$.

Seja $\omega=a \mathrm{~d} x+b \mathrm{~d} y+c \mathrm{~d} z$

$$
\begin{aligned}
& \omega\left(e_{1}\right)=\omega\left(\frac{\partial}{\partial x}-y \frac{\partial}{\partial z}\right)=a-c y=0, \\
& \omega\left(e_{2}\right)=\omega\left(\frac{\partial}{\partial y}+x \frac{\partial}{\partial z}\right)=b+c x=0 .
\end{aligned}
$$

Logo,

$$
\omega=c(y \mathrm{~d} x-x \mathrm{~d} y+\mathrm{d} z) .
$$

Seja $\eta=y \mathrm{~d} x-x \mathrm{~d} y+\mathrm{d} z$. Temos $\omega=c \eta$ e $\left.\eta\right|_{D}=0$,

$$
\begin{aligned}
\mathrm{d} \omega & =\mathrm{d} c \wedge \eta+c \mathrm{~d} \eta \\
\left.\mathrm{d} \omega\right|_{D} & =\left.(\mathrm{d} c \wedge \eta)\right|_{D}+\left.(c \mathrm{~d} \eta)\right|_{D}=c(-2 \mathrm{~d} x \wedge \mathrm{d} y) .
\end{aligned}
$$

Como $\left.\mathrm{d} \omega\right|_{D}=2 \mathrm{~d} A$, temos $c=-1 \mathrm{e} \omega=-y \mathrm{~d} x+x \mathrm{~d} y-\mathrm{d} z$.

Determinemos o campo $\xi$ associado a $\omega$. 
Lembremos que este é o único campo que satisfaz às condições $\omega(\xi)=1$ e $i_{\xi} \mathrm{d} \omega=0$.

Como $\omega\left(-\frac{\partial}{\partial z}\right)=1$ e, $\forall X \in D, \mathrm{~d} \omega\left(-\frac{\partial}{\partial z}, X\right)=2 \mathrm{~d} x \wedge \mathrm{d} y\left(-\frac{\partial}{\partial z}, X\right)=0$, temos $\xi=-\frac{\partial}{\partial z}$.

Na notação do Capítulo 1 temos os referenciais duais $\left\{e_{1}, e_{2}, \xi\right\}$ e $\left\{\theta^{1}, \theta^{2}, \omega\right\}$, onde $\theta^{1}=\mathrm{d} x$ e $\theta^{2}=\mathrm{d} y$.

A métrica é escrita como $g=\left(\theta^{1}\right)^{2}+\left(\theta^{2}\right)^{2}$ e $\mathrm{d} \omega=2 \theta^{1} \wedge \theta^{2}$.

A derivada covariante associada a $\left(\mathbb{R}^{3}, D, g\right), \nabla$, para os campos $e_{1}, e_{2}$ e $\xi$ é escrita como

$$
\nabla e_{i}=\sum_{j} \omega_{i}^{j} e_{j}, \quad 1 \leq i, j \leq 2, \quad \text { e } \quad \nabla \xi=0 .
$$

A primeira equação de estrutura é escrita na forma

$$
\begin{aligned}
& \mathrm{d} \theta^{i}+\sum_{j} \omega_{j}^{i} \wedge \theta^{j}=\omega \wedge \tau^{i}, 1 \leq i, j \leq 2, \\
& \mathrm{~d} \omega=\mathrm{d} \omega .
\end{aligned}
$$

Como $\theta^{1}=\mathrm{d} x$ e $\theta^{2}=\mathrm{d} y$, temos $\mathrm{d} \theta^{1}=\mathrm{d} \theta^{2}=0$. Assim, a primeira equação de estrutura é

$$
\sum_{j} \omega_{j}^{i} \wedge \theta^{j}=\omega \wedge \tau^{i}, 1 \leq i, j \leq 2 .
$$

Pela unicidade de $\omega_{j}^{i}$ e $\tau^{i}$, temos $\omega_{j}^{i}=0, \tau^{i}=0,1 \leq i, j \leq 2$.

Desta maneira, $\nabla e_{i}=0,1 \leq i, j \leq 2$ e $\nabla \xi=0$.

Determinemos agora as geodésicas para o espaço de Heisemberg.

A primeira condição para uma curva

$$
\begin{aligned}
c:[a, b] & \rightarrow \mathbb{R}^{3} \\
t & \rightarrow(x(t), y(t), z(t))
\end{aligned}
$$

ser uma geodésica é que ela seja uma curva tangente à distribuição $D$.

$$
\omega(\dot{c})=(-y \mathrm{~d} x+x \mathrm{~d} y-\mathrm{d} z)(\dot{x}, \dot{y}, \dot{z})=-y \dot{x}+x \dot{y}-\dot{z}=0 .
$$

Através do Teorema 2.3, observando que $\tau=0$, temos $\nabla_{\dot{c}} \dot{c}=k \cdot h(\dot{c})$. 
A transformação $h$ é definida por $\langle h(X), Y\rangle=\mathrm{d} \omega(X, Y)$. Assim,

$$
\begin{aligned}
& \left\langle h\left(e_{1}\right), e_{1}\right\rangle=\mathrm{d} \omega\left(e_{1}, e_{1}\right)=0 \rightarrow h\left(e_{1}\right)=\alpha e_{2}, \\
& \left\langle h\left(e_{2}\right), e_{2}\right\rangle=\mathrm{d} \omega\left(e_{2}, e_{2}\right)=0 \rightarrow h\left(e_{2}\right)=\beta e_{1}, \\
& \left\langle\alpha e_{2}, e_{2}\right\rangle=\mathrm{d} \omega\left(e_{1}, e_{2}\right)=2 \rightarrow \alpha=2, \\
& \left\langle\beta e_{1}, e_{1}\right\rangle=\mathrm{d} \omega\left(e_{2}, e_{1}\right)=-2 \rightarrow \beta=-2 .
\end{aligned}
$$

Portanto, $h\left(e_{1}\right)=2 e_{2}$ e $h\left(e_{2}\right)=-2 e_{1}$.

$$
\begin{aligned}
\dot{c} & =\dot{x} \frac{\partial}{\partial x}+\dot{y} \frac{\partial}{\partial y}+\dot{z} \frac{\partial}{\partial z}=\dot{x} e_{1}+\dot{y} e_{2}+(\dot{x} y-x \dot{y}+\dot{z}) \frac{\partial}{\partial z} \\
& =\dot{x} e_{1}+\dot{y} e_{2}-\omega(\dot{c}) \xi=\dot{x} e_{1}+\dot{y} e_{2}, \\
h(\dot{c}) & =-2 \dot{y} e_{1}+2 \dot{x} e_{2}, \\
\nabla_{\dot{c}} \dot{c} & =\nabla_{\dot{c}}\left(\dot{x} e_{1}+\dot{y} e_{2}\right)=\ddot{x} e_{1}+\dot{x} \nabla_{\dot{c}} e_{1}+\ddot{y} e_{2}+\dot{y} \nabla_{\dot{c}} e_{2}=\ddot{x} e_{1}+\ddot{y} e_{2} .
\end{aligned}
$$

Substituindo em $\nabla_{\dot{c}} \dot{c}=k h(\dot{c})$, temos o sistema

$$
\left\{\begin{array}{l}
\ddot{x}=-2 k \dot{y} \\
\ddot{y}=2 k \dot{x} .
\end{array}\right.
$$

Seja $\lambda=2 k$ e assumamos $|\dot{c}|=1$.

$$
\dddot{x}=-\lambda \ddot{y}=-\lambda^{2} \dot{x}, \quad \dddot{x}+\lambda^{2} \dot{x}=0 .
$$

Se $\lambda \neq 0$, então

$$
\begin{aligned}
& \dot{x}=A \cos (\lambda t+\varphi), \quad A \in \mathbb{R}_{+}, \quad \varphi \in \mathbb{R}, \\
& \dot{y}=\frac{\ddot{x}}{-\lambda}=A \operatorname{sen}(\lambda t+\varphi), \\
& \dot{x}^{2}+\dot{y}^{2}=1 \rightarrow A=1 .
\end{aligned}
$$

Assim,

$$
\dot{x}=\cos (\lambda t+\varphi), \quad \dot{y}=\operatorname{sen}(\lambda t+\varphi),
$$




$$
\begin{aligned}
x & =\frac{1}{\lambda} \operatorname{sen}(\lambda t+\varphi)+x_{0}, \\
y & =-\frac{1}{\lambda} \cos (\lambda t+\varphi)+y_{0}, \\
\dot{z} & =x \dot{y}-y \dot{x}=\left(\frac{1}{\lambda} \operatorname{sen}(\lambda t+\varphi)+x_{0}\right) \operatorname{sen}(\lambda t+\varphi)+\left(\frac{1}{\lambda} \cos (\lambda t+\varphi)-y_{0}\right) \cos (\lambda t+\varphi) \\
& =\frac{1}{\lambda}+x_{0} \operatorname{sen}(\lambda t+\varphi)-y_{0} \cos (\lambda t+\varphi) \\
z & =\frac{1}{\lambda}\left(t-x_{0} \cos (\lambda t+\varphi)-y_{0} \operatorname{sen}(\lambda t+\varphi)\right)+z_{0} .
\end{aligned}
$$

Assim, se $\lambda \neq 0$, as geodésicas parametrizadas pelo comprimento de arco são escritas como

$$
\begin{aligned}
c_{\lambda}(t) & =(x(t), y(t), z(t)), \quad \text { onde } \\
x(t) & =\frac{1}{\lambda} \operatorname{sen}(\lambda t+\varphi)+x_{0}, \\
y(t) & =-\frac{1}{\lambda} \cos (\lambda t+\varphi)+y_{0}, \\
z(t) & =\frac{1}{\lambda}\left(t-x_{0} \cos (\lambda t+\varphi)-y_{0} \operatorname{sen}(\lambda t+\varphi)\right)+z_{0} .
\end{aligned}
$$

Se $\lambda=0$, temos $\ddot{x}=\ddot{y}=0$ e $\dot{x}^{2}+\dot{y}^{2}=1$. Logo,

$$
\begin{aligned}
& \dot{x}=\cos \varphi, \quad \dot{y}=\operatorname{sen} \varphi, \\
& x=t \cos \varphi+x_{0}, \\
& y=t \operatorname{sen} \varphi+y_{0}, \\
& \dot{z}=x \dot{y}-y \dot{x}=\left(t \cos \varphi+x_{0}\right) \operatorname{sen} \varphi-\left(t \operatorname{sen} \varphi+y_{0}\right) \cos \varphi=x_{0} \operatorname{sen} \varphi-y_{0} \cos \varphi, \\
& z=\left(x_{0} \operatorname{sen} \varphi-y_{0} \cos \varphi\right) t+z_{0} .
\end{aligned}
$$

Portanto, se $\lambda=0$, as geodésicas parametrizadas pelo comprimento de arco são escritas na forma $c_{0}(t)=(x(t), y(t), z(t))$, onde

$$
\begin{aligned}
& x(t)=t \cos \varphi+x_{0}, \\
& y(t)=t \operatorname{sen} \varphi+y_{0}, \\
& z(t)=\left(x_{0} \operatorname{sen} \varphi-y_{0} \cos \varphi\right) t+z_{0} .
\end{aligned}
$$


A função

$$
\begin{aligned}
\exp _{p}: D_{p} \times \mathbb{R} & \rightarrow \mathbb{R}^{3} \\
(V, \lambda) & \rightarrow \exp _{p}(V, \lambda)=c_{\lambda}(|V|)
\end{aligned}
$$

onde $c_{\lambda}$ é a geodésica parametrizada pelo comprimento de arco com $c_{\lambda}(0)=p$ e $\dot{c}_{\lambda}(0)=\frac{V}{|V|}$ é chamada aplicação exponencial em $p$.

Vamos determinar a aplicação exponencial na origem, isto é, para $p=0$.

Utilizando coordenadas polares em $D$, escrevemos $V \in D$ como $V=(r, \theta)$.

Para $\lambda \neq 0$, temos

$$
\begin{aligned}
& \dot{c}_{\lambda}(t)=\cos (\lambda t+\varphi) e_{1}+\operatorname{sen}(\lambda t+\varphi) e_{2} \\
& \dot{c}_{\lambda}(0)=\cos \varphi e_{1}+\operatorname{sen} \varphi e_{2}=(1, \varphi), \\
& \frac{V}{|V|}=(1, \theta) .
\end{aligned}
$$

Assim, $\varphi=\theta$.

$$
c_{\lambda}(0)=0, \log 0
$$

$$
\begin{aligned}
& x(0)=\frac{1}{\lambda} \operatorname{sen} \theta+x_{0}=0 \rightarrow x_{0}=-\frac{1}{\lambda} \operatorname{sen} \theta, \\
& y(0)=-\frac{1}{\lambda} \cos \theta+y_{0}=0 \rightarrow y_{0}=\frac{1}{\lambda} \cos \theta, \\
& z(0)=\frac{1}{\lambda}\left(-x_{0} \cos \theta-y_{0} \operatorname{sen} \theta\right)+z_{0}=0 \rightarrow z_{0}=0 .
\end{aligned}
$$

Para $\lambda=0$, temos

$$
\begin{aligned}
& \dot{c}_{0}(0)=\cos \varphi e_{1}+\operatorname{sen} \varphi e_{2}=(1, \varphi)=(1, \theta) \rightarrow \varphi=\theta \\
& c_{0}(0)=0 \rightarrow x_{0}=y_{0}=z_{0}=0 .
\end{aligned}
$$

Assim, a expressão para a aplicação exponencial será

$$
\exp _{0}(r, \theta, 0)=(r \cos \theta, r \operatorname{sen} \theta, 0)
$$




$$
\begin{aligned}
\exp _{0}(r, \theta, \lambda)= & \frac{1}{\lambda}(\operatorname{sen}(\lambda r+\theta)-\operatorname{sen} \theta,-\cos (\lambda r+\theta)+\cos \theta, \\
& \left.r+\frac{1}{\lambda}(\operatorname{sen} \theta \cos (\lambda r+\theta)-\cos \theta \operatorname{sen}(\lambda r+\theta))\right) \\
= & \frac{1}{\lambda}\left(\operatorname{sen}(\lambda r+\theta)-\operatorname{sen} \theta,-\cos (\lambda r+\theta)+\cos \theta, r-\frac{1}{\lambda} \operatorname{sen}(\lambda r)\right) .
\end{aligned}
$$




\section{Exemplo 2: a esfera $S^{3}$}

Neste exemplo vamos utilizar uma identificação do $\mathbb{R}^{4}$ com o $\mathrm{C}^{2}$ de modo que o vetor $\left(x_{1}, x_{2}, x_{3}, x_{4}\right)$ do $\mathbb{R}^{4}$ corresponda ao par $\left(x_{1}+i x_{2}, x_{3}+i x_{4}\right)$ do $\mathbf{C}^{2}$.

Em $\mathrm{C}^{2}$ o produto hermitiano canônico $\langle$, $\rangle$ é definido por $\langle z, w\rangle=z_{1} \bar{w}_{1}+z_{2} \bar{w}_{2}$, onde $z=\left(z_{1}, z_{2}\right)$ e $w=\left(w_{1}, w_{2}\right)$. Seja $z_{1}=x_{1}+i x_{2}, z_{2}=x_{3}+i x_{4}, w_{1}=y_{1}+i y_{2}$ e $w_{2}=y_{3}+i y_{4}$,

$$
\begin{aligned}
\langle z, w\rangle & =\left(x_{1}+i x_{2}\right)\left(y_{1}-i y_{2}\right)+\left(x_{3}+i x_{4}\right)\left(y_{3}-i y_{4}\right) \\
& =x_{1} y_{1}+x_{2} y_{2}+x_{3} y_{3}+x_{4} y_{4}+i\left(x_{2} y_{1}-x_{1} y_{2}+x_{4} y_{3}-x_{3} y_{4}\right) \\
& =((z, w))+i \eta(z, w)
\end{aligned}
$$

onde $(()$,$) é o produto interno do \mathbb{R}^{4}$ e $\eta$ uma forma bilinear anti-simétrica.

Com este produto escalar podemos escrever

$$
\begin{aligned}
S^{3} & =\left\{z \in \mathrm{C}^{2} \mid\langle z, z\rangle=1\right\}, \\
T_{z} S^{3} & =\left\{u \in \mathrm{C}^{2} \mid((z, u))=0\right\} .
\end{aligned}
$$

A distribuição num ponto $z \in S^{3}$ é definida por

$$
D_{z}=\left\{u \in \mathbf{C}^{2} \mid\langle z, u\rangle=0\right\} .
$$

Seja $z^{\perp}=\left(-\bar{z}_{2}, \bar{z}_{1}\right)$, onde $z=\left(z_{1}, z_{2}\right) \in S^{3}$. Assim, $\left\langle z, z^{\perp}\right\rangle=0$ e $D_{z}=\left[z^{\perp}\right]_{\mathbf{C}}$.

$i z \in T_{z} S^{3}$, pois $((z, i z))=0$.

O espaço tangente em $z$ é escrito

$$
T_{z} S^{3}=[i z]_{\mathbb{R}} \oplus D_{z}=[i z]_{\mathbb{R}} \oplus\left[z^{\perp}\right]_{\mathbf{C}}
$$

Nosso objetivo agora é obter a primeira equação de estrutura para $S^{3}$.

Notamos que $\left\{z, z^{\perp}\right\}$ é um referencial unitário pois

$$
\langle z, z\rangle=\left\langle z^{\perp}, z^{\perp}\right\rangle=1 \quad \text { e } \quad\left\langle z, z^{\perp}\right\rangle=0 .
$$


Vamos escrever o referencial canônico $\left\{f_{1}, f_{2}\right\}$ de $\mathrm{C}^{2}$ em função de $z$ e $z^{\perp}$.

$$
\begin{aligned}
z & =z_{1} f_{1}+z_{2} f_{2}, \\
z^{\perp} & =-\bar{z}_{2} f_{1}+\bar{z}_{1} f_{2} .
\end{aligned}
$$

Resolvendo o sistema para $f_{1}$ e $f_{2}$, temos

$$
\begin{aligned}
f_{1} & =\bar{z}_{1} z-z_{2} z^{\perp} \\
f_{2} & =\bar{z}_{2} z+z_{1} z^{\perp} \\
\mathrm{d} z & =\mathrm{d} z_{1} f_{1}+\mathrm{d} z_{2} f_{2} \\
& =\mathrm{d} z_{1}\left(\bar{z}_{1} z-z_{2} z^{\perp}\right)+\mathrm{d} z_{2}\left(\bar{z}_{2} z+z_{1} z^{\perp}\right), \\
& =\left(\bar{z}_{1} \mathrm{~d} z_{1}+\bar{z}_{2} \mathrm{~d} z_{2}\right) z+\left(-z_{2} \mathrm{~d} z_{1}+z_{1} \mathrm{~d} z_{2}\right) z^{\perp}, \\
& =-i\left(\bar{z}_{1} \mathrm{~d} z_{1}+\bar{z}_{2} \mathrm{~d} z_{2}\right)(i z)+\left(-z_{2} \mathrm{~d} z_{1}+z_{1} \mathrm{~d} z_{2}\right) z^{\perp}, \\
\mathrm{d} z^{\perp} & =-\mathrm{d} \bar{z}_{2} f_{1}+\mathrm{d} \bar{z}_{1} f_{2} \\
& =-\mathrm{d} \bar{z}_{2}\left(\bar{z}_{1} z-z_{2} z^{\perp}\right)+\mathrm{d} \bar{z}_{1}\left(\bar{z}_{2} z+z_{1} z^{\perp}\right) \\
& =\left(\bar{z}_{2} \mathrm{~d} \bar{z}_{1}-\bar{z}_{1} \mathrm{~d} \bar{z}_{2}\right) z+\left(z_{1} \mathrm{~d} \bar{z}_{1}+z_{2} \mathrm{~d} \bar{z}_{2}\right) z^{\perp} \\
& =i\left(\bar{z}_{1} \mathrm{~d} \bar{z}_{2}-\bar{z}_{2} \mathrm{~d} \bar{z}_{1}\right)(i z)-i\left(i\left(z_{1} \mathrm{~d} \bar{z}_{1}+z_{2} \mathrm{~d} \bar{z}_{2}\right)\right) z^{\perp} .
\end{aligned}
$$

Seja $\omega=-i\left(\bar{z}_{1} \mathrm{~d} z_{1}+\bar{z}_{2} \mathrm{~d} z_{2}\right)$ e $\varphi=-z_{2} \mathrm{~d} z_{1}+z_{1} \mathrm{~d} z_{2}$

$$
\begin{aligned}
& z_{1} \bar{z}_{1}+z_{2} \bar{z}_{2}=1 \rightarrow z_{1} \mathrm{~d} \bar{z}_{1}+z_{2} \mathrm{~d} \bar{z}_{2}+\bar{z}_{1} \mathrm{~d} z_{1}+\bar{z}_{2} \mathrm{~d} z_{2}=0 \\
& i\left(z_{1} \mathrm{~d} \bar{z}_{1}+z_{2} \mathrm{~d} \bar{z}_{2}\right)=-i\left(\bar{z}_{1} \mathrm{~d} z_{1}+\bar{z}_{2} \mathrm{~d} z_{2}\right) .
\end{aligned}
$$

Portanto, $\omega=\bar{\omega}$ e podemos escrever

$$
\begin{aligned}
& \mathrm{d} z=\omega(i z)+\varphi z^{\perp} \\
& \mathrm{d} z^{\perp}=i \bar{\varphi}(i z)-i \omega z^{\perp} \\
& 0=\mathrm{d}^{2} z=\mathrm{d} \omega(i z)-\omega \wedge \mathrm{d}(i z)+\mathrm{d} \varphi z^{\perp}-\varphi \wedge \mathrm{d} z^{\perp} \\
& =i \mathrm{~d} \omega z-i \omega \wedge\left(\omega(i z)+\varphi z^{\perp}\right)+\mathrm{d} \varphi z^{\perp}-\varphi \wedge\left(i \bar{\varphi}(i z)-i \omega z^{\perp}\right) \\
& =(i \mathrm{~d} \omega+\varphi \wedge \bar{\varphi}) z+(-i \omega \wedge \varphi+\mathrm{d} \varphi+i \varphi \wedge \omega) z^{\perp}
\end{aligned}
$$


Da igualdade acima, obtemos

$$
\begin{aligned}
& \mathrm{d} \varphi-2 i \omega \wedge \varphi=0 \\
& \mathrm{~d} \omega-i \varphi \wedge \bar{\varphi}=0
\end{aligned}
$$

Seja $\theta^{1}=\frac{\varphi+\bar{\varphi}}{2}$ e $\theta^{2}=\frac{\varphi-\bar{\varphi}}{2 i}, \varphi=\theta^{1}+i \theta^{2}$. Assim,

$$
\begin{aligned}
& \mathrm{d} \theta^{1}+i \mathrm{~d} \theta^{2}-2 i \omega \wedge\left(\theta^{1}+i \theta^{2}\right)=0 \\
& \mathrm{~d} \theta^{1}+2 \omega \wedge \theta^{2}=0 \\
& \mathrm{~d} \theta^{2}-2 \omega \wedge \theta^{1}=0 \\
& \mathrm{~d} \omega-i\left(\theta^{1}+i \theta^{2}\right) \wedge\left(\theta^{1}-i \theta^{2}\right)=0 \\
& \mathrm{~d} \omega-2 \theta^{1} \wedge \theta^{2}=0
\end{aligned}
$$

Notamos que d $\left.z\right|_{T_{z} S^{3}}$ é a identidade. Assim, sendo $V \in T_{z} S^{3}$, temos

$$
V=\mathrm{d} z(V)=\omega(V)(i z)+\varphi(V) z^{\perp}=\omega(V)(i z)+\theta^{1}(V) z^{\perp}+\theta^{2}(V)\left(i z^{\perp}\right)
$$

e $\left\{i z, z^{\perp}, i z^{\perp}\right\}$ é uma base real de $T_{z} S^{3}$ cuja base dual é $\left\{\omega, \theta^{1}, \theta^{2}\right\}$.

A métrica sobre $S^{3}$ induzida do $\mathbb{R}^{4}$ é $g_{1}=(\omega)^{2}+\left(\theta^{1}\right)^{2}+\left(\theta^{2}\right)^{2}$, pois os vetores $i z, z^{\perp}$ e $i z^{\perp}$ são ortonormais em $\mathbb{R}^{4}$.

Em $D$ temos a métrica $g=\left(\theta^{1}\right)^{2}+\left(\theta^{2}\right)^{2}$.

$\left(S^{3}, D, g\right)$ é uma variedade sub-riemanniana de contato onde, utilizando a notação do Capítulo 1 , temos $e_{1}=z^{\perp}, e_{2}=i z^{\perp}, \xi=i z$ e $\omega$ é a forma de contato.

A primeira equação de estrutura é

$$
\begin{aligned}
& \mathrm{d} \theta^{1}+2 \omega \wedge \theta^{2}=0, \\
& \mathrm{~d} \theta^{2}-2 \omega \wedge \theta^{1}=0, \\
& \mathrm{~d} \omega-2 \theta^{1} \wedge \theta^{2}=0 .
\end{aligned}
$$

Da terceira igualdade, $\mathrm{d} \omega=2 \mathrm{~d} A$. 
A derivada covariante associada à estrutura sub-riemanniana é escrita

$$
\begin{aligned}
\nabla e_{1} & =-2 \omega e_{2}, \\
\nabla e_{2} & =2 \omega e_{1}, \\
\nabla \xi & =0 .
\end{aligned}
$$

Observamos que $\nabla$ é compatível com a estrutura complexa de $D_{z}$, pois

$$
i \nabla z^{\perp}=i\left(-2 \omega e_{2}\right)=-2 i \omega\left(i z^{\perp}\right)=2 \omega e_{1}=\nabla i z^{\perp}
$$

e, por linearidade, $\forall \alpha \in \mathrm{C}, \nabla \alpha z^{\perp}=\alpha \nabla z^{\perp}$.

Conhecendo a derivada covariante, vamos determinar as geodésicas de $S^{3}$.

Seja $c:\left[a_{1}, b_{1}\right] \rightarrow S^{3}$

$$
t \rightarrow c(t)=z(t)=\left(z_{1}(t), z_{2}(t)\right)
$$

Um campo ao longo de $c$ é escrito

$$
X(t)=a(t) z^{\perp}+b(t)(i z)
$$

onde $a:\left[a_{1}, b_{1}\right] \rightarrow \mathrm{C}$ e $b:\left[a_{1}, b_{1}\right] \rightarrow \mathbb{R}$,

$$
\begin{aligned}
\nabla_{\dot{c} X} & =\frac{\mathrm{d} a}{\mathrm{~d} t} z^{\perp}+a \nabla_{\dot{c}} z^{\perp}+\frac{\mathrm{d} b}{\mathrm{~d} t}(i z)+b \nabla_{\dot{c}}(i z) \\
& =\left(\frac{\mathrm{d} a}{\mathrm{~d} t}-2 i a \omega(\dot{c})\right) z^{\perp}+\frac{\mathrm{d} b}{\mathrm{~d} t}(i z), \\
\dot{c} & =\dot{z}_{1} f_{1}+\dot{z}_{2} f_{2}=\omega(\dot{c})(i z)+\varphi(\dot{c}) z^{\perp} .
\end{aligned}
$$

A curva $c$ é uma geodésica se e somente se $\dot{c} \in D$ e $\nabla_{\dot{c}} \dot{c}=\lambda h(\dot{c}), \lambda \in \mathbb{R}$.

$$
\begin{aligned}
& \dot{c} \in D \leftrightarrow \omega(\dot{c})=0, \\
& \dot{c}=\varphi(\dot{c}) z^{\perp}=\left(-z_{2} \mathrm{~d} z_{1}+z_{1} \mathrm{~d} z_{2}\right)\left(\dot{z}_{1}, \dot{z}_{2}\right) z^{\perp} \\
& =\left(-z_{2} \dot{z}_{1}+z_{1} \dot{z}_{2}\right) z^{\perp}=a z^{\perp} .
\end{aligned}
$$

Assim, fazendo $X=\dot{c}$ na expressão de $\nabla_{\dot{c}} X$, temos

$$
\nabla_{\dot{c}} \dot{c}=\frac{\mathrm{d} a}{\mathrm{~d} t} z^{\perp}=\frac{\mathrm{d}}{\mathrm{d} t}\left(-z_{2} \dot{z}_{1}+z_{1} \dot{z}_{2}\right) z^{\perp}=\left(-z_{2} \ddot{z}_{1}+z_{1} \ddot{z}_{2}\right) z^{\perp} .
$$


Pelo mesmo argumento utilizado no espaço de Heisemberg; temos $h\left(e_{1}\right)=2 e_{2}$ e $h\left(e_{2}\right)=$ $-2 e_{1}$. Assim, $\forall V \in D_{z}, h(V)=2 i V$.

$$
\begin{aligned}
& \nabla_{\dot{c} \dot{c}}=\lambda h(\dot{c})=2 i \lambda \dot{c}=2 i \lambda a z^{\perp}, \\
& \frac{\mathrm{d} a}{\mathrm{~d} t} z^{\perp}=2 i \lambda a z^{\perp} \rightarrow a(t)=K e^{2 i \lambda t}, K \in \mathrm{C} .
\end{aligned}
$$

Como $\dot{c}=a z^{\perp}$, temos $\left(\dot{z}_{1}, \dot{z}_{2}\right)=a\left(-z_{2}, z_{1}\right)$.

$$
\begin{aligned}
& \left\{\begin{array}{l}
\dot{z}_{1}=-a \bar{z}_{2} \\
\dot{z}_{2}=a \bar{z}_{1}
\end{array}\right. \\
& \ddot{z}_{1}=-\frac{\mathrm{d} a}{\mathrm{~d} t} \bar{z}_{2}-a \dot{\bar{z}}_{2}=-\frac{\mathrm{d} a}{\mathrm{~d} t}\left(\frac{\dot{z_{1}}}{-a}\right)-a \bar{a} z_{1}=2 i \lambda \dot{z}_{1}-|a|^{2} z_{1}, \\
& \ddot{z}_{1}-2 i \lambda \dot{z}_{1}+|a|^{2} z_{1}=0 \text {. }
\end{aligned}
$$

Vamos considerar geodésicas parametrizadas pelo comprimento de arco.

$$
\begin{aligned}
& \langle\dot{z}, \dot{z}\rangle=a \bar{a}\left\langle z^{\perp}, z^{\perp}\right\rangle=|a|^{2}=|K|^{2}=1, \\
& \ddot{z}_{1}-2 i \lambda \dot{z}_{1}+z_{1}=0, \\
& z_{1}=K_{1} e^{i \beta_{1} t}+K_{2} e^{i \beta_{2} t},
\end{aligned}
$$

onde $\beta_{1}=\lambda+\sqrt{1+\lambda^{2}}, \beta_{2}=\lambda-\sqrt{1+\lambda^{2}}$ e $K_{1}, K_{2} \in \mathrm{C}$.

$$
\begin{aligned}
& \dot{z}_{1}=i \beta_{1} K_{1} e^{i \beta_{1} t}+i \beta_{2} K_{2} e^{i \beta_{2} t}, \\
& K \cdot \bar{K}=1, \quad 1 / \bar{K}=K, \\
& z_{2}=\frac{\bar{z}_{1}}{-\bar{a}}=\frac{-i \beta_{1} \bar{K}_{1} e^{-i \beta_{1} t}-i \beta_{2} \bar{K}_{2} e^{-i \beta_{2} t}}{-\bar{K} e^{-2 i \lambda t}} \\
& =i K\left(\beta_{1} \bar{K}_{1} e^{i\left(2 \lambda-\beta_{1}\right) t}+\beta_{2} \bar{K}_{2} e^{i\left(2 \lambda-\beta_{2}\right) t}\right) \\
& =i K\left(\beta_{1} \bar{K}_{1} e^{i \beta_{2} t}+\beta_{2} \bar{K}_{2} e^{i \beta_{1} t}\right) .
\end{aligned}
$$

As constantes $K_{1}, K_{2}$ e $K$ são determinadas a partir dos valores $z(0)=\left(z_{1}(0), z_{2}(0)\right)$ e $\dot{z}(0)=\left(\dot{z}_{1}(0), \dot{z}_{2}(0)\right)$.

Como $K e^{2 i \lambda t}=-z_{2} \dot{z}_{1}+z_{1} \dot{z}_{2}=\left\langle\dot{z}, z^{\perp}\right\rangle$, temos $K=\left\langle\dot{z}(0), z^{\perp}(0)\right\rangle$.

$$
\begin{aligned}
z_{1}(0) & =K_{1}+K_{2}, \\
z_{2}(0) & =i K \beta_{1} \bar{K}_{1}+i K \beta_{2} \bar{K}_{2} .
\end{aligned}
$$


Resolvendo o sistema para $K_{1}$ e $K_{2}$, obtemos

$$
K_{1}=\frac{\beta_{2} z_{1}(0)-i K \overline{z_{2}(0)}}{\beta_{2}-\beta_{1}}, \quad K_{2}=\frac{\beta_{1} z_{1}(0)-i K \overline{z_{2}(0)}}{\beta_{1}-\beta_{2}} .
$$

Como $\dot{z} \in D, \frac{\mathrm{d}}{\mathrm{d} t}\langle z(t), z(t)\rangle=\langle\dot{z}(t), z(t)\rangle+\langle z(t), \dot{z}(t)\rangle=0$, assim $\langle z(t), z(t)\rangle=$ $\langle z(0), z(0)\rangle=1$ e $c(t) \in S^{3}$.

As geodésicas em $S^{3}$ parametrizadas pelo comprimento de arco, com condições iniciais $z(0)=\left(z_{1}(0), z_{2}(0)\right)$ e $\dot{z}(0)=\left(\dot{z}_{1}(0), \dot{z}_{2}(0)\right)$ são escritas

$$
\begin{aligned}
& c_{\lambda}:\left[a_{1}, b_{1}\right] \rightarrow S^{3} \\
& t \rightarrow c(t)=\left(z_{1}(t), z_{2}(t)\right) \\
& z_{1}(t)=K_{1} e^{i \beta_{1} t}+K_{2} e^{i \beta_{2} t} \\
& z_{2}(t)=i K\left(\beta_{1} \bar{K}_{1} e^{i \beta_{2} t}+\beta_{2} \bar{K}_{2} e^{i \beta_{1} t}\right),
\end{aligned}
$$

onde

$$
\begin{aligned}
\beta_{1} & =\lambda+\sqrt{1+\lambda^{2}}, \quad \beta_{2}=\lambda-\sqrt{1+\lambda^{2}}, \\
K & =\left\langle\dot{z}(0), z^{\perp}(0)\right\rangle, \\
K_{1} & =\frac{\beta_{2} z_{1}(0)-i K \overline{z_{2}(0)}}{\beta_{2}-\beta_{1}} \quad \text { e } \quad K_{2}=\frac{\beta_{1} z_{1}(0)-i K \overline{z_{2}(0)}}{\beta_{1}-\beta_{2}} .
\end{aligned}
$$

Vamos determinar a aplicação exponencial no ponto $(0, i)$.

$$
\begin{aligned}
& D_{(0, i)}=[(i, 0)]_{\mathbf{C}}, \\
& V \in D_{(0, i)} \leftrightarrow V=v(i, 0), v \in \mathbf{C}, \\
& \|V\|=\|v(i, 0)\|=|v|, \\
& \frac{V}{\|V\|}=\frac{v(i, 0)}{|v|}, \\
& z(0)=(0, i), \quad \dot{z}(0)=\frac{V}{\|V\|}=\frac{v}{|v|}(i, 0), \\
& K=\left\langle\dot{z}(0), z^{\perp}(0)\right\rangle=\left\langle\frac{v}{|v|}(i, 0),(i, 0)\right\rangle=\frac{v}{|v|}, \\
& K_{1}=\frac{-i\left(\frac{v}{|v|}\right)(-i)}{\beta_{2}-\beta_{1}}=\frac{v}{2|v| \sqrt{1+\lambda^{2}}}, \\
& K_{2}=\frac{-v}{2|v| \sqrt{1+\lambda^{2}}}=-K_{1} .
\end{aligned}
$$


A aplicação exponencial em $(0, i)$ é escrita

$$
\begin{aligned}
\exp _{(0, i)} D_{(0, i)} \times \mathbb{R} & \rightarrow S^{3} \\
(v(i, 0), \lambda) & \rightarrow \exp _{(0, i)}(v(i, 0), \lambda)=c_{\lambda}(|v|)
\end{aligned}
$$

$\exp _{(0, i)}(v(i, 0), \lambda)=\left(z_{1}, z_{2}\right)$, onde

$$
\begin{aligned}
z_{1} & =\frac{v}{2|v| \sqrt{1+\lambda^{2}}}\left(e^{i\left(\lambda+\sqrt{1+\lambda^{2}}\right)|v|}-e^{i\left(\lambda-\sqrt{1+\lambda^{2}}\right)|v|}\right) \quad \mathrm{e} \\
z_{2} & =i \frac{v}{|v|} \frac{\bar{v}}{2|v| \sqrt{1+\lambda^{2}}}\left(\beta_{1} e^{i \beta_{2}|v|}-\beta_{2} e^{i \beta_{1}|v|}\right) \\
& =\frac{i}{2 \sqrt{1+\lambda^{2}}}\left(\left(\lambda+\sqrt{1+\lambda^{2}}\right) e^{i\left(\lambda-\sqrt{1+\lambda^{2}}\right)|v|}-\left(\lambda-\sqrt{1+\lambda^{2}}\right) e^{i\left(\lambda+\sqrt{1+\lambda^{2}}\right)|v|}\right) .
\end{aligned}
$$




\section{Exemplo 3: a quádrica $\mathbb{Q}^{3}$}

Como no exemplo anterior, identificamos o $\mathbb{R}^{4} \operatorname{com}$ o $\mathrm{C}^{2}$. Em $\mathrm{C}^{2}$ definimos $\langle$,$\rangle por$

$$
\langle z, w\rangle=z_{1} \bar{w}_{1}-z_{2} \bar{w}_{2}
$$

onde $z=\left(z_{1}, z_{2}\right), w=\left(w_{1}, w_{2}\right)$.

Seja $z_{1}=x_{1}+i x_{2}, z_{2}=x_{3}+i x_{4}, w_{1}=y_{1}+i y_{2}$ e $w_{2}=y_{3}+i y_{4}$.

$$
\begin{aligned}
\langle z, w\rangle & =\left(x_{1}+i x_{2}\right)\left(y_{1}-i y_{2}\right)-\left(x_{3}+i x_{4}\right)\left(y_{3}-i y_{4}\right) \\
& =x_{1} y_{1}+x_{2} y_{2}-x_{3} y_{3}-x_{4} y_{4}+i\left(x_{2} y_{1}-x_{1} y_{2}-x_{4} y_{3}+x_{3} y_{4}\right) \\
& =((z, w))+i \eta(z, w), \\
\mathbb{Q}^{3} & =\left\{z \in \mathrm{C}^{2} \mid\langle z, z\rangle=-1\right\}, \\
T_{z} \mathbb{Q}^{3} & =\left\{u \in \mathrm{C}^{2} \mid((z, u))=0\right\} .
\end{aligned}
$$

A distribuição num ponto $z \in \mathbb{Q}^{3}$ é definida por

$$
D_{z}=\left\{u \in \mathbf{C}^{2} \mid\langle z, u\rangle=0\right\} .
$$

Seja $z^{\perp}=\left(\bar{z}_{2}, \bar{z}_{1}\right)$, onde $z=\left(z_{1}, z_{2}\right) \in \mathbb{Q}^{3}$. Assim, $\left\langle z, z^{\perp}\right\rangle=0$ e $D_{z}=\left[z^{\perp}\right]_{\mathbf{C}}$.

$-i z \in T_{z} S^{3}$, pois $((z, i z))=0$.

$\mathrm{O}$ espaço tangente em $z$ é escrito $T_{z} \mathbb{Q}^{3}=[-i z]_{\mathbb{R}} \oplus\left[z^{\perp}\right]_{\mathbf{C}}$.

Nosso objetivo agora é obter a primeira equação de estrutura para $\mathbb{Q}^{3}$.

Vamos escrever o referencial canônico $\left\{f_{1}, f_{2}\right\}$ de $C^{2}$ em função de $z$ e $z^{\perp}$.

$$
\begin{aligned}
z & =z_{1} f_{1}+z_{2} f_{2}, \\
z^{\perp} & =\bar{z}_{2} f_{1}+\bar{z}_{1} f_{2} .
\end{aligned}
$$

Resolvendo o sistema para $f_{1}$ e $f_{2}$, temos

$$
f_{1}=-\bar{z}_{1} z+z_{2} z^{\perp}
$$




$$
\begin{aligned}
f_{2} & =\bar{z}_{2} z-z_{1} z^{\perp} \\
\mathrm{d} z & =\mathrm{d} z_{1} f_{1}+\mathrm{d} z_{2} f_{2} \\
& =\mathrm{d} z_{1}\left(-\bar{z}_{1} z+z_{2} z^{\perp}\right)+\mathrm{d} z_{2}\left(\bar{z}_{2} z-z_{1} z^{\perp}\right) \\
& =\left(-\bar{z}_{1} \mathrm{~d} z_{1}+\bar{z}_{2} \mathrm{~d} z_{2}\right) z+\left(z_{2} \mathrm{~d} z_{1}-z_{1} \mathrm{~d} z_{2}\right) z^{\perp} \\
& =i\left(-\bar{z}_{1} \mathrm{~d} z_{1}+\bar{z}_{2} \mathrm{~d} z_{2}\right)(-i z)+\left(z_{2} \mathrm{~d} z_{1}-z_{1} \mathrm{~d} z_{2}\right) z^{\perp}, \\
\mathrm{d} z^{\perp} & =\mathrm{d} \bar{z}_{2} f_{1}+\mathrm{d} \bar{z}_{1} f_{2} \\
& =\mathrm{d} \bar{z}_{2}\left(-\bar{z}_{1} z+z_{2} z^{\perp}\right)+\mathrm{d} \bar{z}_{1}\left(\bar{z}_{2} z-z_{1} z^{\perp}\right) \\
& =\left(-\bar{z}_{1} \mathrm{~d} \bar{z}_{2}+\bar{z}_{2} \mathrm{~d} \bar{z}_{1}\right) z+\left(z_{2} \mathrm{~d} \bar{z}_{2}-z_{1} \mathrm{~d} \bar{z}_{1}\right) z^{\perp} \\
& =i\left(-\bar{z}_{1} \mathrm{~d} \bar{z}_{2}+\bar{z}_{2} \mathrm{~d} \bar{z}_{1}\right)(-i z)+i\left(-i\left(z_{2} \mathrm{~d} \bar{z}_{2}-z_{1} \mathrm{~d} \bar{z}_{1}\right)\right) z^{\perp} .
\end{aligned}
$$

Seja $\omega=i\left(-\bar{z}_{1} \mathrm{~d} z_{1}+\bar{z}_{2} \mathrm{~d} z_{2}\right)$ e $\varphi=z_{2} \mathrm{~d} z_{1}-z_{1} \mathrm{~d} z_{2}$,

$$
\begin{aligned}
& z_{1} \bar{z}_{1}-z_{2} \bar{z}_{2}=-1 \rightarrow z_{1} \mathrm{~d} \bar{z}_{1}-z_{2} \mathrm{~d} \bar{z}_{2}+\bar{z}_{1} \mathrm{~d} z_{1}-\bar{z}_{2} \mathrm{~d} z_{2}=0, \\
& i\left(-\bar{z}_{1} \mathrm{~d} z_{1}+\bar{z}_{2} \mathrm{~d} z_{2}\right)=-i\left(-z_{1} \mathrm{~d} \bar{z}_{1}+z_{2} \mathrm{~d} \bar{z}_{2}\right) .
\end{aligned}
$$

Portanto, $\omega=\bar{\omega}$ e podemos escrever

$$
\begin{aligned}
& \mathrm{d} z=\omega(-i z)+\varphi z^{\perp} \\
& \mathrm{d} z^{\perp}=i \bar{\varphi}(-i z)+i \omega z^{\perp}, \\
& 0=\mathrm{d}^{2} z=\mathrm{d} \omega(-i z)-\omega \wedge \mathrm{d}(-i z)+\mathrm{d} \varphi z^{\perp}-\varphi \wedge \mathrm{d} z^{\perp} \\
& =-i \mathrm{~d} \omega z+i \omega \wedge\left(\omega(-i z)+\varphi z^{\perp}\right)+\mathrm{d} \varphi z^{\perp}-\varphi \wedge\left(i \bar{\varphi}(i z)+i \omega z^{\perp}\right) \\
& =(-i \mathrm{~d} \omega-\varphi \wedge \bar{\varphi}) z+(i \omega \wedge \varphi+\mathrm{d} \varphi-i \varphi \wedge \omega) z^{\perp} .
\end{aligned}
$$

Da igualdade acima, obtemos

$$
\begin{aligned}
& \mathrm{d} \varphi+2 i \omega \wedge \varphi=0, \\
& \mathrm{~d} \omega-i \varphi \wedge \bar{\varphi}=0 .
\end{aligned}
$$


Seja $\theta^{1}=\frac{\varphi+\bar{\varphi}}{2}$ e $\theta^{2}=\frac{\varphi-\bar{\varphi}}{2 i}, \varphi=\theta^{1}+i \theta^{2}$. Assim,

$$
\begin{aligned}
& \mathrm{d} \theta^{1}+i \mathrm{~d} \theta^{2}+2 i \omega \wedge\left(\theta^{1}+i \theta^{2}\right)=0 \\
& \mathrm{~d} \theta^{1}-2 \omega \wedge \theta^{2}=0 \\
& \mathrm{~d} \theta^{2}+2 \omega \wedge \theta^{1}=0 \\
& \mathrm{~d} \omega-i\left(\theta^{1}+i \theta^{2}\right) \wedge\left(\theta^{1}-i \theta^{2}\right)=0 \\
& \mathrm{~d} \omega-2 \theta^{1} \wedge \theta^{2}=0 .
\end{aligned}
$$

$\forall V \in T_{z} \mathbb{Q}^{3}, \mathrm{~d} z(V)=V=\omega(V)(-i z)+\varphi(V) z^{\perp}=\omega(V)(-i z)+\theta^{1}(V) z^{\perp}+\theta^{2}(V)\left(i z^{\perp}\right)$.

Em $D_{z}$ temos a base ortonormal $\left\{z^{\perp}, i z^{\perp}\right\}$ com base dual $\left\{\theta^{1}, \theta^{2}\right\}$. A métrica em $D$ é $g=\left(\theta^{1}\right)^{2}+\left(\theta^{2}\right)^{2}$.

$\left(\mathbb{Q}^{3}, D, g\right)$ é uma variedade sub-riemanniana de contato onde, utilizando a notação do Capítulo 1, temos $e_{1}=z^{\perp}, e_{2}=i z^{\perp}, \xi=-i z$ e $\omega$ é a forma de contato.

A primeira equação de estrutura é

$$
\begin{aligned}
& \mathrm{d} \theta^{1}-2 \omega \wedge \theta^{2}=0, \\
& \mathrm{~d} \theta^{2}+2 \omega \wedge \theta^{1}=0, \\
& \mathrm{~d} \omega-2 \theta^{1} \wedge \theta^{2}=0 \quad(\mathrm{~d} \omega=2 \mathrm{~d} A) .
\end{aligned}
$$

A derivada covariante associada à estrutura sub-riemanniana é escrita

$$
\begin{aligned}
\nabla e_{1} & =2 \omega e_{2}, \\
\nabla e_{2} & =-2 \omega e_{1}, \\
\nabla \xi & =0 .
\end{aligned}
$$

$\nabla$ é compatível com a estrutura complexa de $D_{z}$, pois

$$
i \nabla z^{\perp}=i\left(2 \omega e_{2}\right)=-2 \omega e_{1}=\nabla e_{2}=\nabla i z^{\perp}
$$

e, por linearidade, $\forall \alpha \in \mathrm{C}, \nabla \alpha z^{\perp}=\alpha \nabla z^{\perp}$. 
Vamos determinar as geodésicas em $\mathbb{Q}^{3}$.

Seja

$$
\begin{aligned}
& c:\left[a_{1}, b_{1}\right] \rightarrow \mathbb{Q}^{3} \\
& t \rightarrow c(t)=z(t)=\left(z_{1}(t), z_{2}(t)\right) \\
& \dot{c}=\dot{z}_{1} f_{1}+\dot{z}_{2} f_{2}=\omega(\dot{c})(-i z)+\varphi(\dot{c}) z^{\perp} .
\end{aligned}
$$

A curva $c$ é uma geodésica se e somente se $\dot{c} \in D$ e $\nabla_{\dot{c}} \dot{c}=\lambda h(\dot{c}), \lambda \in \mathbb{R}$.

$$
\begin{aligned}
& \dot{c}=\varphi(\dot{c}) z^{\perp}=a z^{\perp}, \\
& \nabla_{\dot{c}} \dot{c}=\frac{\mathrm{d} a}{\mathrm{~d} t} z^{\perp}+a \nabla_{\dot{c}} z^{\perp}=\frac{\mathrm{d} a}{\mathrm{~d} t} z^{\perp}+2 a \omega(\dot{c})(i z)=\frac{\mathrm{d} a}{\mathrm{~d} t} z^{\perp}, \\
& \nabla_{\dot{c}} \dot{c}=\lambda h(\dot{c})=2 i \lambda \dot{c}=2 i \lambda a z^{\perp}, \\
& \frac{\mathrm{d} a}{\mathrm{~d} t}=2 i \lambda a \rightarrow a=K e^{2 i \lambda t}, K \in \mathrm{C}, \\
& \dot{c}=a z^{\perp} \rightarrow\left(\dot{z}_{1}, \dot{z}_{2}\right)=a\left(\bar{z}_{2}, \bar{z}_{1}\right), \\
& \left\{\begin{array}{l}
\dot{z}_{1}=a \bar{z}_{2} \\
\dot{z}_{2}=a \bar{z}_{1}
\end{array}\right. \\
& \ddot{z}_{1}=\frac{\mathrm{d} a}{\mathrm{~d} t} \bar{z}_{2}+a \dot{\bar{z}}_{2}=\frac{\mathrm{d} a}{\mathrm{~d} t}\left(\frac{\dot{z}_{1}}{a}\right)+a \bar{a} z_{1}=2 i \lambda \dot{z}_{1}+|a|^{2} z, \\
& \ddot{z}_{1}-2 i \lambda \dot{z}_{1}-|a|^{2} z=0 .
\end{aligned}
$$

Vamos considerar geodésicas parametrizadas pelo comprimento de arco.

$$
\begin{aligned}
& g(\dot{c}, \dot{c})=\langle\dot{z}, \dot{z}\rangle=a \bar{a}\left\langle z^{\perp}, z^{\perp}\right\rangle=|a|^{2}=|K|^{2}=1, \\
& \ddot{z}_{1}-2 i \lambda \dot{z}_{1}-z=0, \\
& z_{1}=K_{1} e^{i \beta_{1} t}+K_{2} e^{i \beta_{2} t}, \text { onde } \beta_{1}=\lambda+\sqrt{\lambda^{2}-1}, \beta_{2}=\lambda-\sqrt{\lambda^{2}-1} \\
& \text { e } K_{1}, K_{2} \in \mathrm{C}, \text { se } \lambda \neq \pm 1, \\
& z_{1}=K_{1} e^{i t}+t K_{2} e^{i t}, \text { onde } K_{1}, K_{2} \in \mathrm{C}, \text { se } \lambda=1, \\
& z_{1}=K_{1} e^{-i t}+t K_{2} e^{-i t}, \quad \text { onde } K_{1}, K_{2} \in \mathrm{C}, \text { se } \lambda=-1, \\
& \dot{z}_{1}=i \beta_{1} K_{1} e^{i \beta_{1} t}+i \beta_{2} K_{2} e^{i \beta_{2} t}, \quad \text { se } \lambda \neq \pm 1,
\end{aligned}
$$




$$
\begin{aligned}
& \dot{z}_{1}=i K_{1} e^{i t}+K_{2} e^{i t}+i t K_{2} e^{i t}, \quad \text { se } \lambda=1, \\
& \dot{z}_{1}=-i K_{1} e^{-i t}+K_{2} e^{-i t}-i t K_{2} e^{-i t}, \quad \text { se } \lambda=-1, \\
& z_{2}=\frac{\bar{z}_{1}}{\bar{a}} \\
& z_{2}=\frac{-i \bar{\beta}_{1} \bar{K}_{1} e^{-i \bar{\beta}_{1} t}-i \bar{\beta}_{2} \bar{K}_{2} e^{-i \bar{\beta}_{2} t}}{\bar{K} e^{-2 i \lambda t}} \\
& z_{2}=-i K\left(\bar{\beta}_{1} \bar{K}_{1} e^{i \bar{\beta}_{2} t}+\bar{\beta}_{2} \bar{K}_{2} e^{i \bar{\beta}_{1} t}\right), \text { se } \lambda \neq \pm 1, \\
& z_{2}=K\left(\left(-i \bar{K}_{1}+\bar{K}_{2}\right) e^{i t}-i t \bar{K}_{2} e^{i t}\right), \quad \text { se } \lambda=1, \\
& z_{2}=K\left(\left(i \bar{K}_{1}+\bar{K}_{2}\right) e^{-i t}+i t \bar{K}_{2} e^{-i t}\right), \quad \text { se } \lambda=-1 .
\end{aligned}
$$

As constantes $K_{1}, K_{2}$ e $K$ são determinadas a partir dos valores $z(0)=\left(z_{1}(0), z_{2}(0)\right)$ e $\dot{z}(0)=\left(\dot{z}_{1}(0), \dot{z}_{2}(0)\right)$

$$
\begin{aligned}
K e^{2 i \lambda t} & =\varphi(\dot{c})=z_{2} \dot{z}_{1}-z_{1} \dot{z}_{2}=\left\langle\dot{z}, z^{\perp}\right\rangle, \\
K & =\left\langle\dot{z}(0), z^{\perp}(0)\right\rangle .
\end{aligned}
$$

Se $\lambda \neq \pm 1$,

$$
\begin{aligned}
& z_{1}(0)=K_{1}+K_{2} \\
& z_{2}(0)=-i K\left(\bar{\beta}_{1} \bar{K}_{1}+\bar{\beta}_{2} \bar{K}_{2}\right) .
\end{aligned}
$$

Resolvendo o sistema para $K_{1}$ e $K_{2}$, temos

$$
K_{1}=\frac{\beta_{2} z_{1}(0)+i K \overline{z_{2}(0)}}{\beta_{2}-\beta_{1}}, \quad K_{2}=\frac{\beta_{1} z_{1}(0)+i K \overline{z_{2}(0)}}{\beta_{1}-\beta_{2}} .
$$

Se $\lambda=1$,

$$
\begin{aligned}
& z_{1}(0)=K_{1} \\
& z_{2}(0)=K\left(-i \bar{K}_{1}+\bar{K}_{2}\right) .
\end{aligned}
$$

Portanto, $K_{1}=z_{1}(0), K_{2}=K \overline{z_{2}(0)}-i z_{1}(0)$. 
Se $\lambda=-1$,

$$
\begin{aligned}
& z_{1}(0)=K_{1}, \\
& z_{2}(0)=K\left(i \bar{K}_{1}+\bar{K}_{2}\right) .
\end{aligned}
$$

Portanto, $K_{1}=z_{1}(0), K_{2}=K \overline{z_{2}(0)}+i z_{1}(0)$.

Como $\dot{z} \in D, \frac{\mathrm{d}}{\mathrm{d} t}\langle z(t), z(t)\rangle=\langle\dot{z}(t), z(t)\rangle+\langle z(t), \dot{z}(t)\rangle=0 . \quad \operatorname{Assim}\langle z(t), z(t)\rangle=$ $\langle z(0), z(0)\rangle=-1$ e $c(t) \in \mathbb{Q}^{3}$.

As geodésicas em $Q^{3}$ parametrizadas pelo comprimento de arco, com condições iniciais $z(0)=\left(z_{1}(0), z_{2}(0)\right)$ e $\dot{z}(0)=\left(\dot{z}_{1}(0), \dot{z}_{2}(0)\right)$ são escritas

$$
\begin{aligned}
& c_{\lambda}:\left[a_{1}, b_{1}\right] \rightarrow \mathbb{Q}^{3} \\
& t \rightarrow c(t)=\left(z_{1}(t), z_{2}(t)\right) \\
& z_{1}(t)=K_{1} e^{i \beta_{1} t}+K_{2} e^{i \beta_{2} t} \\
& z_{2}(t)=-i K\left(\bar{\beta}_{1} \bar{K}_{1} e^{i \bar{\beta}_{2} t}+\bar{\beta}_{2} \bar{K}_{2} e^{i \bar{\beta}_{1} t}\right),
\end{aligned}
$$

onde

$$
\begin{aligned}
& \beta_{1}=\lambda+\sqrt{\lambda^{2}-1}, \quad \beta_{2}=\lambda-\sqrt{\lambda^{2}-1} \\
& K=\left\langle\dot{z}(0), z^{\perp}(0)\right\rangle, \\
& K_{1}=\frac{\beta_{2} z_{1}(0)+i K \overline{z_{2}(0)}}{\beta_{2}-\beta_{1}} \quad \text { e } \quad K_{2}=\frac{\beta_{1} z_{1}(0)+i K \overline{z_{2}(0)}}{\beta_{1}-\beta_{2}}, \quad \text { se } \lambda \neq \pm 1, \\
& z_{1}(t)=\left(K_{1}+t K_{2}\right) e^{i t}, \\
& z_{2}(t)=K\left(-i \bar{K}_{1}+\bar{K}_{2}(1-i t)\right) e^{i t},
\end{aligned}
$$

onde $K=\left\langle\dot{z}(0), z^{\perp}(0)\right\rangle, K_{1}=z_{1}(0)$ e $K_{2}=K \overline{z_{2}(0)}-i z_{1}(0)$, se $\lambda=1$,

$$
\begin{aligned}
& z_{1}(t)=\left(K_{1}+t K_{2}\right) e^{-i t} \\
& z_{2}(t)=K\left(i \bar{K}_{1}+\bar{K}_{2}(1+i t)\right) e^{-i t}
\end{aligned}
$$

onde $K=\left\langle\dot{z}(0), z^{\perp}(0)\right\rangle, K_{1}=z_{1}(0)$ e $K_{2}=K \overline{z_{2}(0)}+i z_{1}(0)$, se $\lambda=-1$. 
Vamos determinar a aplicação exponencial no ponto $(0, i)$.

$$
\begin{aligned}
& V \in D_{(0, i)} \leftrightarrow V=v(-i, 0), v \in \mathrm{C}, \\
& \|V\|=\|v(-i, 0)\|=|v|, \\
& \frac{V}{\|V\|}=\frac{v(-i, 0)}{|v|}, \\
& z(0)=(0, i), \quad \dot{z}(0)=\frac{V}{\|V\|}=\frac{v}{|v|}(-i, 0), \\
& K=\left\langle\dot{z}(0), z^{\perp}(0)\right\rangle=\left\langle\frac{v}{|v|}(-i, 0),(-i, 0)\right\rangle=\frac{v}{|v|} .
\end{aligned}
$$

Se $\lambda \neq \pm 1$,

$$
K_{1}=\frac{i K(-i)}{\beta_{2}-\beta_{1}}=\frac{-v}{2|v| \sqrt{\lambda^{2}-1}}=-K_{2} .
$$

Se $\lambda=1$,

$$
K_{1}=0, \quad K_{2}=K(-i)=\frac{-i v}{|v|}
$$

Se $\lambda=-1$,

$$
K_{1}=0, \quad K_{2}=K(-i)=\frac{-i v}{|v|} .
$$

A aplicação exponencial em $(0, i)$ é escrita

$$
\begin{aligned}
\exp _{(0, i)}: D_{(0, i)} \times \mathbb{R} & \rightarrow \mathbb{Q}^{3} \\
(v(-i, 0), \lambda) & \rightarrow \exp _{(0, i)}(v(-i, 0), \lambda)=c_{\lambda}(|v|)
\end{aligned}
$$

$\exp _{(0, i)}(v(-i, 0), \lambda)=\left(z_{1}, z_{2}\right)$, onde

$$
\begin{aligned}
& z_{1}=\frac{v}{2|v| \sqrt{\lambda^{2}-1}}\left(-e^{i\left(\lambda+\sqrt{\lambda^{2}-1}\right)|v|}+e^{i\left(\lambda-\sqrt{\lambda^{2}-1}\right)|v|}\right) \\
& z_{2}=\frac{i}{2 \overline{\sqrt{\lambda^{2}-1}}}\left(\overline{\left(\lambda+\sqrt{\lambda^{2}-1}\right)} e^{i\left(\lambda-\sqrt{\lambda^{2}-1}\right)}|v|-\overline{\left(\lambda-\sqrt{\lambda^{2}-1}\right)} e^{i\left(\overline{\left.\lambda+\sqrt{\lambda^{2}+1}\right)}|v|\right.}\right) \\
& \text { se } \lambda \neq \pm 1 \\
& z_{1}=-i v e^{i|v|} \\
& z_{2}=(i+|v|) e^{i|v|}, \quad \text { se } \lambda=1, \\
& z_{1}=-i v e^{-i|v|} \\
& z_{2}=(i-|v|) e^{-i|v|}, \text { se } \lambda=-1 \text {. }
\end{aligned}
$$




\section{Referências}

[1] CAMACHO, C. \& LINS NETO. Teoria geométrica de folheações. Projeto Euclides, IMPA, Rio de Janeiro.

[2] CARMO, M.P. DO Differential geometry of curves and surfaces. Englewood Cliffs, Prentice Hall, 1976.

[3] CARMO, M.P. DO Geometria Riemanniana. Projeto Euclides, IMPA, Rio de Janeiro, 1988.

[4] CHERN, S.S. \& HAMILTON, R.S. On Riemannian metrics adapted to three-dim. contact manifolds. Lec. Notes in Math., 1111, 279-305.

[5] DINIZ, M.M. Variedades sub-riemannianas de contato de dim. 3. Mestrado IMEUSP, 1996.

[6] GODBILLON, C. Geometrie différentielle et mécanique analytique. Herman, Paris, 1969.

[7] HSU, L. Calculus of variations via the Griffiths formalism. J. Diff. Geom., 36 (1992) 551-589.

[8] KOBAYASHI, S. \& NOMIZU, K. Foundations of differential geometry. v.1. Interscience (Wiley), New York, 1963.

[9] MONTGOMERY, R. A survey of singular curves in sub-riemannian geometry. $J$. Dyn. Contr. Sys., 1 (1995) 49-90.

[10] RUMIN, M. Formes differentielles sur les variétés de contact. J. Diff. Geom., 39(2) (1994) 281-330.

[11] STRICHARTS, R.S. Sub-riemannian geometry. J. Diff. Geom., 24 (1986) 221-263.

[12] TIKHONOV, A.N.; VASIL'EVA, A.B. \& SVESHNIKOV, A.G. Differential equations. Springer-Verlag.

[13] VERSHIK, A.M.; GERSHKOVICH, V.Ya. Nonholonomic dynamical systems, geometry of distributions and variational problems. In: ARNOLD, V.I.; NOVIKOV, S.P. (Eds.). Dynamical systems VII: Integrable systems nonholonomic dynamical systems. Berlin: Springer-Verlag, 1994, p.4-81. 138p. (Encyclopaedia of Mathematical Sciences, v.16)

[14] WARNER, F.W. Foundations of differential manifolds and Lie groups. Scott, Foresman, Genview, 1971. 